\title{
A high-order 3-D spectral-element method for the forward modelling and inversion of gravimetric data-Application to the western Pyrenees
}

\author{
Roland Martin, ${ }^{1}$ Sébastien Chevrot, ${ }^{1}$ Dimitri Komatitsch, ${ }^{2}$ Lucia Seoane, ${ }^{1}$ \\ Hannah Spangenberg, ${ }^{1}$ Yi Wang, ${ }^{1}$ Grégory Dufréchou, ${ }^{1}$ Sylvain Bonvalot ${ }^{1}$ \\ and Sean Bruinsma ${ }^{3}$ \\ ${ }^{1}$ Laboratoire de Géosciences Environnement Toulouse GET, CNRS UMR 5563, Observatoire Midi-Pyrénées, Université Paul Sabatier, \\ 14 avenue Édouard Belin, F-31400Toulouse, France.E-mail: roland.martin@get.omp.eu \\ ${ }^{2}$ LMA, CNRS UPR 7051, Aix-Marseille University, Centrale Marseille, F-13453 Marseille, France \\ ${ }^{3}$ CNES, F-31400 Toulouse, France
}

Accepted 2017 January 10. Received 2016 December 14; in original form 2016 July 28

\begin{abstract}
S UMMAR Y
We image the internal density structure of the Pyrenees by inverting gravity data using an a priori density model derived by scaling a $V_{p}$ model obtained by full waveform inversion of teleseismic $P$-waves. Gravity anomalies are computed via a 3-D high-order finite-element integration in the same high-order spectral-element grid as the one used to solve the wave equation and thus to obtain the velocity model. The curvature of the Earth and surface topography are taken into account in order to obtain a density model as accurate as possible. The method is validated through comparisons with exact semi-analytical solutions. We show that the spectral-element method drastically accelerates the computations when compared to other more classical methods. Different scaling relations between compressional velocity and density are tested, and the Nafe-Drake relation is the one that leads to the best agreement between computed and observed gravity anomalies. Gravity data inversion is then performed and the results allow us to put more constraints on the density structure of the shallow crust and on the deep architecture of the mountain range.
\end{abstract}

Key words: Numerical solutions; Inverse theory; Gravity anomalies and Earth structure.

\section{INTRODUCTION}

Seismic tomography is commonly used to obtain images of the Earth's interior. Among the different tomographic approaches, full waveform inversion (FWI) is a promising technique for improved resolution of tomographic images in spite of its high computational cost (Tarantola \& Valette 1982; Lailly 1983; Tarantola 1984; Tromp et al. 2005, 2008; Virieux \& Operto 2009; Fichtner 2010; Monteiller et al. 2013, 2015). In theory, FWI has the potential to resolve simultaneously not only the compressional and shear velocities but also density. However, density is not generally well imaged with seismic data alone (Wang et al. 2016). This suggests that adding gravimetric data to seismic data could potentially improve our capacity to image the density distribution in the lithosphere. Furthermore, empirical correlations between compressional wave speed and density are now well documented (Birch 1961; Ludwig et al. 1970; Gardner et al. 1974; Brocher 2005). Such relations carry important a priori information that can help us to better constrain the solution of the joint inversion problem in the lithosphere. In this study, we invert seismic data collected in the Pyrenees region during the PYROPE and IBERARRAY temporary experiments (Chevrot et al. 2014, 2015). Our ultimate goal is to obtain models of density and seismic velocities in order to constrain the composition and thermal state of the lithosphere. We focus more particularly on the western PYROPE transect (Chevrot et al. 2015) and construct an a priori density model by scaling the $V_{p}$ model obtained by FWI (Monteiller et al. 2015; Wang et al. 2016) to density. Starting from this $a$ priori model we perform an iterative inversion of Bouguer gravity anomalies measured on the topography.

Different forward modelling approaches have been used to compute gravity anomalies (Barnett 1976; Coggon 1976; Okabe 1979; Gotze \& Lahmeyer 1988; Pohánka 1988; Yao \& Changli 2007). Analytical integrations inside prismatic bodies (Bhattacharyya 1964; Nagy 1966; Plouff 1976; Bhattacharyya 1980; Nagy et al. 2000), polygons with arbitrary shapes (Talwani et al. 1959; Talwani \& Ewing 1960; Talwani 1965), polyhedra (Tsoulis 2012), tesseroids (Uieda et al. 2010, 2011, 2016) or fast multipole methods (Casenave et al. 2016) have for instance been introduced. Building from these different forward modelling approaches, a wide variety of inversion techniques has been 
developed in the last decade. For instance, the semi-analytical prismatic body approach has been used to image complex structures such as sulphide ore bodies or salt domes (Dubey et al. 2014; Dubey \& Tiwari 2016). Parallel inversion of gravity data in southwest Ghana (Perrouty et al. 2012; Martin et al. 2013) has been performed from an initial a priori model constructed with the Geomodeller software package (Lajaunie et al. 1997; Calcagno et al. 2008; Guillen et al. 2008), the choice of the a priori model being a crucial issue. Nagihara \& Hall (2001) applied a simulated-annealing global optimization technique to invert synthetic gravity data computed for a salt dome model. The shape of the dome at depth was constrained using 3-D inverse modelling. Simulated annealing has also been applied by Roy et al. (2005) to estimate water-sediment and sediment-basement interfaces. Zhang et al. (2004) have imaged the crustal structure of central Taiwan using a stochastic parallel genetic algorithm and an a priori model derived from traveltime tomography. René (1986) developed gravity inversion by iteratively applying open, reject and fill criteria within a modelling procedure based on initially assigned prismatic body density contrasts and a shape-of-anomaly fill criterion. Uieda \& Barbosa (2012a,b) performed 3-D gravity inversion by using a shape-of-anomaly data misfit and $L^{2}$-norm data misfit to better delineate geological structures. Recently, Nava-Flores et al. (2016) applied stochastic simulated annealing inversion to determine salt dome structures trapped inside sediments in the Gulf of Mexico, using an a priori model obtained by pre-stack migration of seismic data.

In this study, we develop an efficient and accurate high-order spectral-element method (SEM) to compute gravity anomalies on the same grid as the one used to solve the elastic wave equation (Komatitsch \& Tromp 1999; Peter et al. 2011). An advantage of the SEM is that topography can be handled naturally. After comparing the solutions computed with the SEM using different polynomial degrees to exact analytical solutions, we demonstrate that we can use the same polynomial order to handle both seismic and gravity problems. Furthermore we show that the SEM drastically accelerates the computations by at least a factor of around 40 when compared to more classical semi-analytical techniques. We then test different a priori density anomaly models constructed with different $V_{p}$ to $\rho$ scaling relations. Effects of topography and ellipticity of the Earth are added to compute solutions that are as accurate as possible. An inversion process is then performed using a least-square technique that is accelerated via parallelization of the code TOMOFAST3D described in Martin et al. (2013). Furthermore, a depth-weighting operator similar to the one introduced by Li \& Oldenburg (1998) and Pilkington (1997) for gravity or magnetic data is applied to the sensitivity matrix and to the density model. This weighting enhances the sensitivity to density perturbations at depth. A wavelet compression of the sensitivity matrices is introduced in our procedure to accelerate it further (as in Li \& Oldenburg (2003) for magnetic data inversion). We apply this inversion technique to the data of a southwest-northeast profile across the Mauléon basin north of the Pyrenees (France), in which strong Bouguer anomalies are observed.

\section{COMPUTATION OF BOUGUER GRAVITY ANOMALIES}

\subsection{Principle}

The gravity force is the resultant of the gravitational and centrifugal forces. In this study, since we work at the regional scale, we do not take into account the rotation of the Earth. Thus, in the absence of centrifugal forces, the gravitational potential $\Phi$ at a given position $\mathbf{r}$ caused by an arbitrary density distribution $\rho$ in a 3-D Earth domain $\Omega$ is given by

$\Phi(\mathbf{r})=G \int_{\Omega} \frac{\rho\left(\mathbf{r}^{\prime}\right)}{\left\|\mathbf{r}-\mathbf{r}^{\prime}\right\|} \mathrm{d} \mathbf{r}^{\prime}$

where $\mathbf{r}^{\prime}$ is the position of an elementary density cell volume, and $G$ the universal constant of gravity equal to $6.672 \times 10^{-11} \mathrm{~m}^{3} \mathrm{~kg}^{-1} \mathrm{~s}^{-2}$. The components of the gravity acceleration resulting from the distribution of masses in a Cartesian coordinate system $(\xi, \eta, \zeta)$ at an observational point $(x, y, z)$ located at the surface are given by

$g_{x}(x, y, z)=\frac{\partial \Phi}{\partial x}=G \int_{\Omega} \rho \frac{(x-\xi)}{\left[(x-\xi)^{2}+(y-\eta)^{2}+(z-\zeta)^{2}\right]^{3 / 2}} \mathrm{~d} \xi \mathrm{d} \eta \mathrm{d} \zeta$

$g_{y}(x, y, z)=\frac{\partial \Phi}{\partial y}=G \int_{\Omega} \rho \frac{(y-\eta)}{\left[(x-\xi)^{2}+(y-\eta)^{2}+(z-\zeta)^{2}\right]^{3 / 2}} \mathrm{~d} \xi \mathrm{d} \eta \mathrm{d} \zeta$

$g_{z}(x, y, z)=\frac{\partial \Phi}{\partial z}=G \int_{\Omega} \rho \frac{(z-\zeta)}{\left[(x-\xi)^{2}+(y-\eta)^{2}+(z-\zeta)^{2}\right]^{3 / 2}} \mathrm{~d} \xi \mathrm{d} \eta \mathrm{d} \zeta$.

In this paper we will only consider the vertical component of the gravity field $g_{z}$.

Let us also note that the second-order gravity tensor $g=\left(G_{x x}, G_{x y}, G_{x z}, G_{y y}, G_{y z}\right)$ can be computed following the same philosophy.

\subsection{Computation of density kernels}

\subsubsection{The classical semi-analytical approach}

Let us consider an Earth model discretized into a set of prisms with rectangular sections. The gravity kernel $S$ can be computed using the semi-analytical formulae given by Blakely (1995) and implemented in his GBOX routines. This gravity kernel expresses the contribution of 
each voxel (i.e. each mesh cell volume) to the gravity field. The vertical component of the gravity anomaly $\Delta g_{z}$ due to a density anomaly $\Delta \rho$ is then

$\Delta g_{z}(x, y, z)=\sum_{i=1}^{2} \sum_{j=1}^{2} \sum_{k=1}^{2} S_{i, j, k}(x, y, z) \Delta \rho_{i, j, k}$,

where

$S_{i, j, k}(x, y, z)=G \mu_{i, j, k}\left[z_{k} \tan ^{-1}\left(\frac{x_{i} y_{j}}{z_{k} R_{i, j, k}}\right)-x_{i} \log \left(R_{i, j, k}+y_{j}\right)-y_{j} \log \left(R_{i, j, k}+x_{i}\right)\right]$,

with $R_{i, j, k}=\sqrt{x_{i}^{2}+y_{j}^{2}+z_{k}^{2}}, \mu_{i, j, k}=(-1)^{i+j+k}, x_{i}=x-\xi_{i}, y_{j}=y-\eta_{j}, z_{k}=z-\zeta_{k}, i, j, k=1$ or 2 , and $\left(\xi_{i}, \eta_{j}, \zeta_{k}\right)$ being the coordinates of the elemental cell vertices.

\subsubsection{The finite-element approach}

If one wants to consider more complicated geometries for the integration by taking into account the topography of the free surface or of internal discontinuities, one can introduce a finite-element integration. The idea is to use the same numerical integration rules as in finite-element methods. Furthermore, integration rules based on Gauss quadrature to compute eq. (4) will avoid the computation of logarithms and arctangents, without loss of accuracy. Numerical integrals are performed on collocated or non-collocated points using quadrature weights. Here we make the choice of using a spectral-element integration approach in space, in which each cube is sampled at Gauss-Lobatto-Legendre (GLL) points using high-order Lagrange interpolants. At any observation point $(x, y, z)$ above topography, the three components of $\mathbf{g}$ are given by

$g_{x}(x, y, z)=G \int_{\Omega} \rho \frac{(x-\xi)}{\left[(x-\xi)^{2}+(y-\eta)^{2}+(z-\zeta)^{2}\right]^{3 / 2}} J \mathrm{~d} \alpha \mathrm{d} \beta \mathrm{d} \psi$

$g_{y}(x, y, z)=G \int_{\Omega} \rho \frac{(y-\eta)}{\left[(x-\xi)^{2}+(y-\eta)^{2}+(z-\zeta)^{2}\right]^{3 / 2}} J \mathrm{~d} \alpha \mathrm{d} \beta \mathrm{d} \psi$

$g_{z}(x, y, z)=G \int_{\Omega} \rho \frac{(z-\zeta)}{\left[(x-\xi)^{2}+(y-\eta)^{2}+(z-\zeta)^{2}\right]^{3 / 2}} J \mathrm{~d} \alpha \mathrm{d} \beta \mathrm{d} \psi$

where $J$ is the local Jacobian of the bijective mapping (assuming that the Jacobian is non-singular and thus invertible) of the reference cube ( $\alpha$, $\beta, \psi) \in[-1,1] \times[-1,1] \times[-1,1]$ to a given deformed cube $(\xi, \eta, \zeta) \in \Omega$. Then, after discretization and integration on elementary deformed cubes, the particular case of the vertical component of gravity anomaly (along the $z$ direction) is computed as

$\Delta g_{z}(x, y, z)=G \sum_{l, i, j, k} \Delta \rho_{i j k}^{l} \frac{\left(z-\zeta_{i j k}^{l}\right)}{\left[\left(x-\xi_{i j k}^{l}\right)^{2}+\left(y-\eta_{i j k}^{l}\right)^{2}+\left(z-\zeta_{i j k}^{l}\right)^{2}\right]^{3 / 2}} J_{i j k}^{l} \omega_{i} \omega_{j} \omega_{k} d \alpha d \beta d \psi$

where $l=1, N, i=1, N_{\alpha}, j=1, N_{\beta}, k=1, N_{\psi} . \Delta \rho$ is the density anomaly, $N$ is the number of elementary cell volumes, $N_{\alpha}, N_{\beta}$ and $N_{\psi}$ are the number of collocation GLL points associated to each cell along the axes $\alpha, \beta$ and $\psi$, and the $\omega_{l}$ coefficients (with $l=i, j$ or $k$ ) are the quadrature weights. The $x$ and $y$ components can be computed in the same way.

If a single-point Gauss-quadrature integration is chosen, one has $\omega_{m}=1$ for $m=i, j$ or $k$ and $N_{\alpha}=N_{\beta}=N_{\psi}=1, d \xi=d \eta=d \gamma=1$. The effect of density in block $l$ on the gravity anomaly at position $\left(i_{D}, j_{D}, k_{D}\right)$ located at point $(x, y, z)$ of the surface can then be defined as:

$\Delta g_{z, l}^{i_{D}, j_{D}, k_{D}}=G \sum_{i, j, k} \Delta \rho_{i j k}^{l} \frac{\left(z-\zeta_{i j k}^{l}\right)}{\left[\left(x-\xi_{i j k}^{l}\right)^{2}+\left(y-\eta_{i j k}^{l}\right)^{2}+\left(z-\zeta_{i j k}^{l}\right)^{2}\right]^{3 / 2}} J_{i j k}^{l} \omega_{i} \omega_{j} \omega_{k}$

where $i=1, N_{\alpha}, j=1, N_{\beta}$ and $k=1, N_{\psi}$. One can then write (11) in a more compact form:

$\Delta g_{z}(x, y, z)=\sum_{l=1, N} g_{l}^{i_{D}, j_{D}, k_{D}}=G \sum_{l=1, N} M^{l} \Delta \rho^{l}=G \sum_{l=1, N} \sum_{i=1, N_{\alpha}} \sum_{j=1, N_{\beta}} \sum_{k=1, N_{\psi}} M_{i j k}^{l} \Delta \rho_{i j k}^{l}$,

where $M^{l}$ is the local gravity mass matrix, $M_{i j k}^{l}$ the element of $M^{l}$ assigned to each local GLL point, and $\rho_{i j k}^{l}$ the density anomaly assigned to each local GLL point. The second-order tensor $g=\left(G_{x x}, G_{x y}, G_{x z}, G_{y y}, G_{y z}\right)$ can be computed in a way similar to the algebraic calculations of gravity anomalies in eqs (10) and (11). Finally, as can be seen in eq. (12), the anomaly gravity field can thus be computed by the matrix-vector product:

$\Delta g_{z}=S \Delta \rho$,

where $S=G M$ is the global gravity kernel. 


\section{COMPARISON BETWEEN SEMI-ANALYTICAL AND FINITE-ELEMENT COMPUTATIONS OF BOUGUER GRAVITY ANOMALIES}

Let us now validate and assess the accuracy of our spectral-element integration method by computing synthetic Bouguer anomalies predicted for different density distributions: a single sphere, one hundred spheres, a density model defined by different depth-dependent density gradients, and a prior density model provided by FWI in the more realistic case of the western Pyrenees. The results obtained based on the SEM approach are compared to those obtained with the classical semi-analytical (GBOX) method approach of Blakely (1995) as well as to exact analytical solutions.

\subsection{Synthetic cases}

\subsubsection{Sphere distribution}

We first consider the simple case of a $3 \mathrm{~km}$ radius sphere centred at $25 \mathrm{~km}$ depth in the middle of a $220 \mathrm{~km} \times 10 \mathrm{~km} \times 50 \mathrm{~km}$ computational domain. The average spacing between points in the computational domain is approximately $0.91,0.5$ and $0.84 \mathrm{~km}$ along the $x, y$ and $z$ axes, respectively. The density inside the sphere is $2700 \mathrm{~kg} \mathrm{~m}^{-3}$. The number of cells is 240,20 and 60 along the $x, y$ and $z$ axes, respectively for the GBOX/Blakely semi-analytical method, while the number of GLL points for the SEM is 241, 21 and 65 along each axis (corresponding to $60 \times 5 \times 16$ spectral finite elements and a slightly smaller $z$-spacing of $0.78 \mathrm{~km}$ ). In Fig. 1 , one can see that for a similar number of

(A)

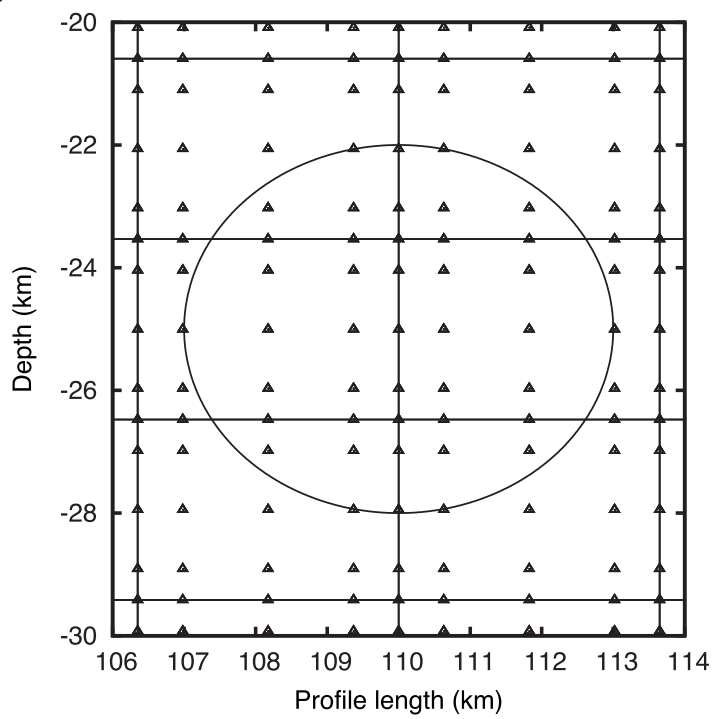

(C)

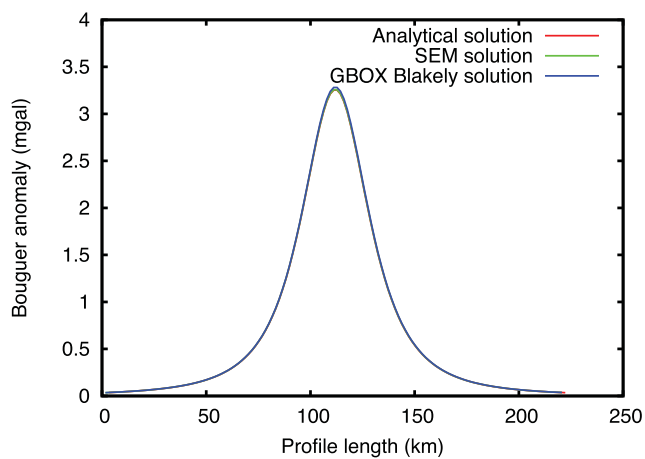

(B)

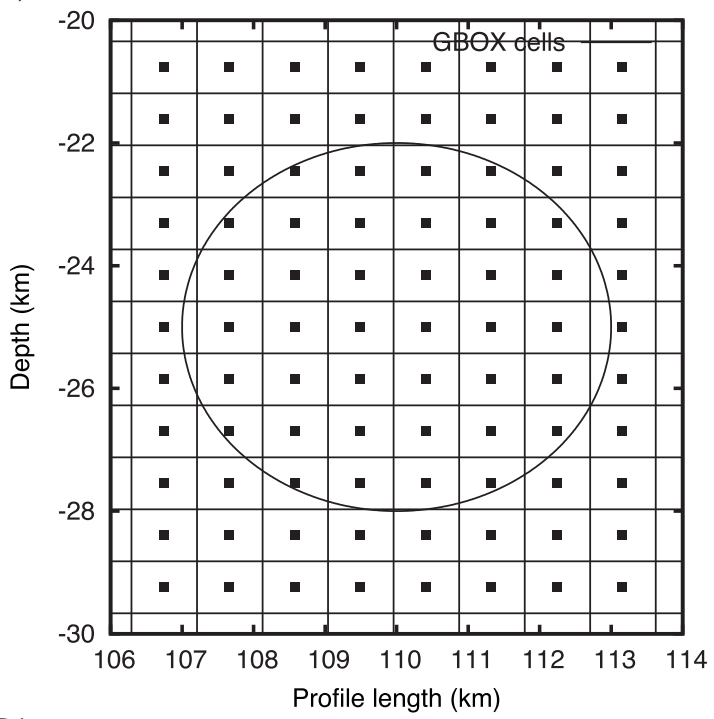

(D)

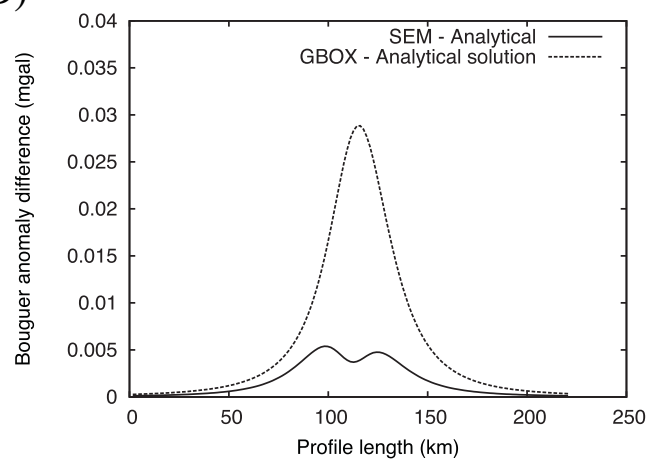

Figure 1. We show (A) the GLL points mesh (triangles) and the edges of the spectral elements as well as (B) the GBOX cells and their centres to illustrate the discretization differences between the SEM and GBOX for a single sphere case. The discretized density values inside the sphere are assigned to the GLL points located inside the sphere, while they are assigned to each entire cell whose centre is inside the sphere in the case of GBOX. (C) Comparison of Bouguer gravity anomalies computed with the semi-analytical and spectral-element integration methods for the case of a single $3 \mathrm{~km}$ radius sphere located at $25 \mathrm{~km}$ depth. The numerical solution is almost identical to the exact analytical solution. In the SEM approach, five GLL collocation points are used along each spatial direction of a 3-D spectral element. (D) Errors with respect to the analytical solution. Larger errors are obtained when using the semi-analytical GBOX approach due to the fact that one has fewer prismatic bodies included in the sphere, while more discretization GLL points are located inside in the SEM approach. The computations are 40 times faster with the SEM compared to GBOX. 

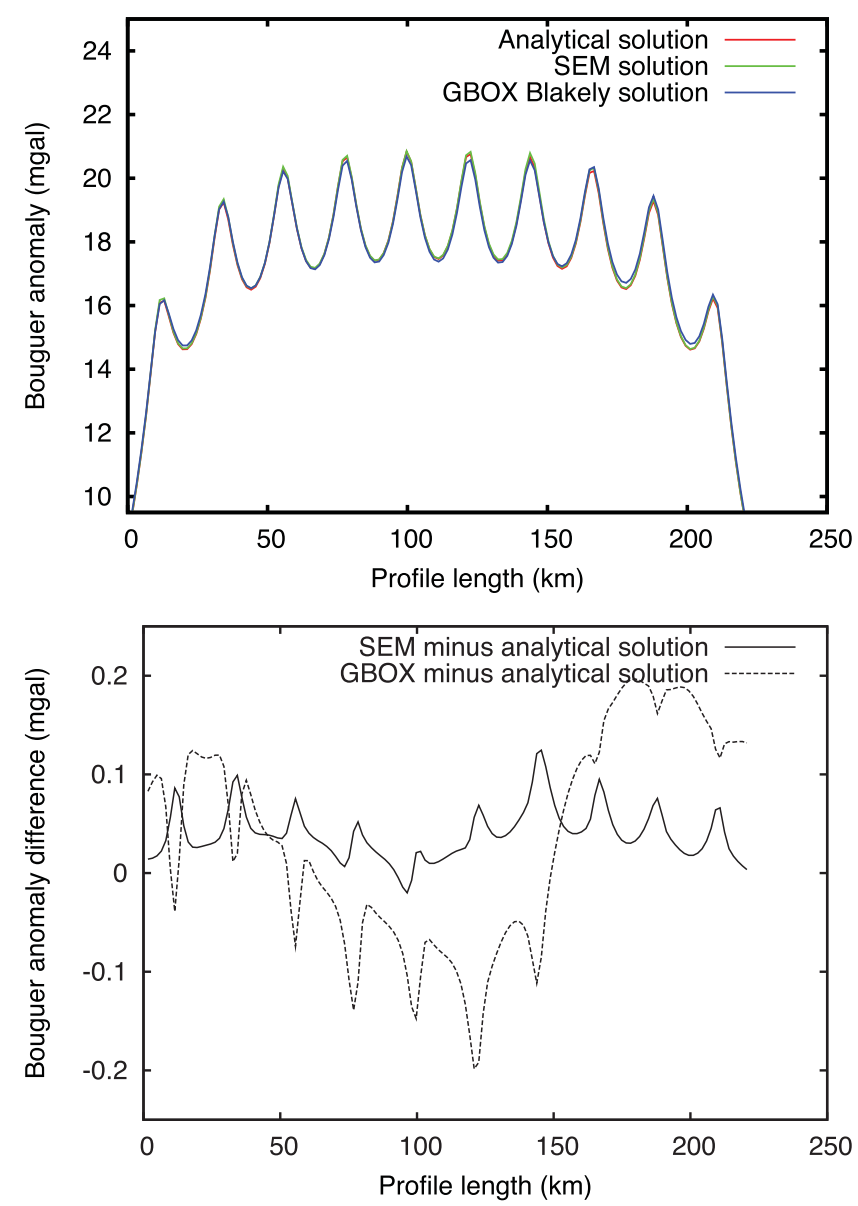

Figure 2. Top: comparison of gravity anomalies in the case of 100 spheres located in a vertical plane when using semi-analytical or spectral-element integration methods. The numerical solutions are almost identical to the exact analytical solution. In the SEM approach, five GLL collocation points are used in each spatial direction of a 3-D spectral element. Bottom: larger errors are obtained when using the semi-analytical GBOX approach due to the fact that one has fewer prismatic bodies included in a sphere, while one has more discretization GLL points inside in the SEM approach. Maximum errors of around 1.1 per cent in the GBOX case and less than 0.6 per cent in the SEM case are obtained.

discretization points the semi-analytical solution is slightly less accurate than the SEM solution when compared to the exact solution given by

$\Delta g_{z}(x, z)=G \sum_{l=1, N_{s}} \pi R_{l}^{3} \Delta \rho_{l} \frac{z-z_{l}}{3\left[\left(x-x_{l}\right)^{2}+\left(z-z_{l}\right)\right]^{3 / 2}}$

where $N_{s}$ is the number of spheres, $\Delta \rho_{l}, R_{l}, x_{l}$ and $z_{l}$ are respectively the density, the radius, the distance and the depth of the centre of $l$ th sphere. The four vertices of each spectral element in the 2-D vertical plane are shown (there are eight such vertices in the 3-D calculations) and the number of GLL points is similar to the number of GBOX cells. Solutions are very similar but the main difference between the two methods lies in the computational speed (around $8 \mathrm{~s}$ for SEM and $318 \mathrm{~s}$ for GBOX on a current $3 \mathrm{GHz}$ PC). We thus obtain a computing acceleration of around 40 with SEM compared to GBOX. The reason is that GBOX involves logarithm and arctangent functions which have a high computational cost, while in the SEM only simple computations such as sums and multiplications are needed.

For the same $220 \mathrm{~km} \times 10 \mathrm{~km} \times 50 \mathrm{~km}$ computational domain, we now consider a more complex medium with a density model defined by 10 lines of 10 spheres spaced by $22 \mathrm{~km}$ along the $x$ longitudinal direction, and by $4 \mathrm{~km}$ along the depth direction down to a depth of $50 \mathrm{~km}$, the centre of each sphere being located at a depth of $5 \mathrm{~km}$ for the first line and at a depth of $41 \mathrm{~km}$ for the last line of spheres. All the lines start at $x=10 \mathrm{~km}$. The centres of all the spheres are located in the same vertical plane. The density is constant inside each sphere but varies linearly between the first/top line and the last/bottom line, from 250 to $2050 \mathrm{~kg} \mathrm{~m}^{-3}$ according to $\rho_{l}=50(1+4 l) \mathrm{kg} \mathrm{m}^{-3}, l=1-10 \mathrm{being}$ the line number. At line $l$ all the spheres have the same radius equal to $2+(l-1) / 9 \mathrm{~km}(2 \mathrm{~km}$ on the first/top line and $3 \mathrm{~km}$ on the last/bottom line). The number of GBOX/Blakely cells is $200 \times 20 \times 60$ while the number of GLL points for the SEM is $209 \times 21 \times 65$ (corresponding to $52 \times 5 \times 16$ spectral finite elements). We use an average spatial resolution of $4.2 \mathrm{~km}$ along $x, 2 \mathrm{~km}$ along $y$ and $3.125 \mathrm{~km}$ along $z$ between the spectral-element control (vertex) nodes and an average spacing around $1.05 \mathrm{~km}$ along $x, 0.5 \mathrm{~km}$ along $y$ and $0.78 \mathrm{~km}$ along $z$ between the GLL points, for a number of points equal to 5 in each spatial direction of each spectral element. Similar spacings are taken for GBOX. The semi-analytical solution is slightly less accurate than the SEM solution when compared to the exact solution computed by summing the anomalies produced by each individual sphere (Fig. 2). The error is around 2 per cent for the semi-analytical approach, while the SEM gives 

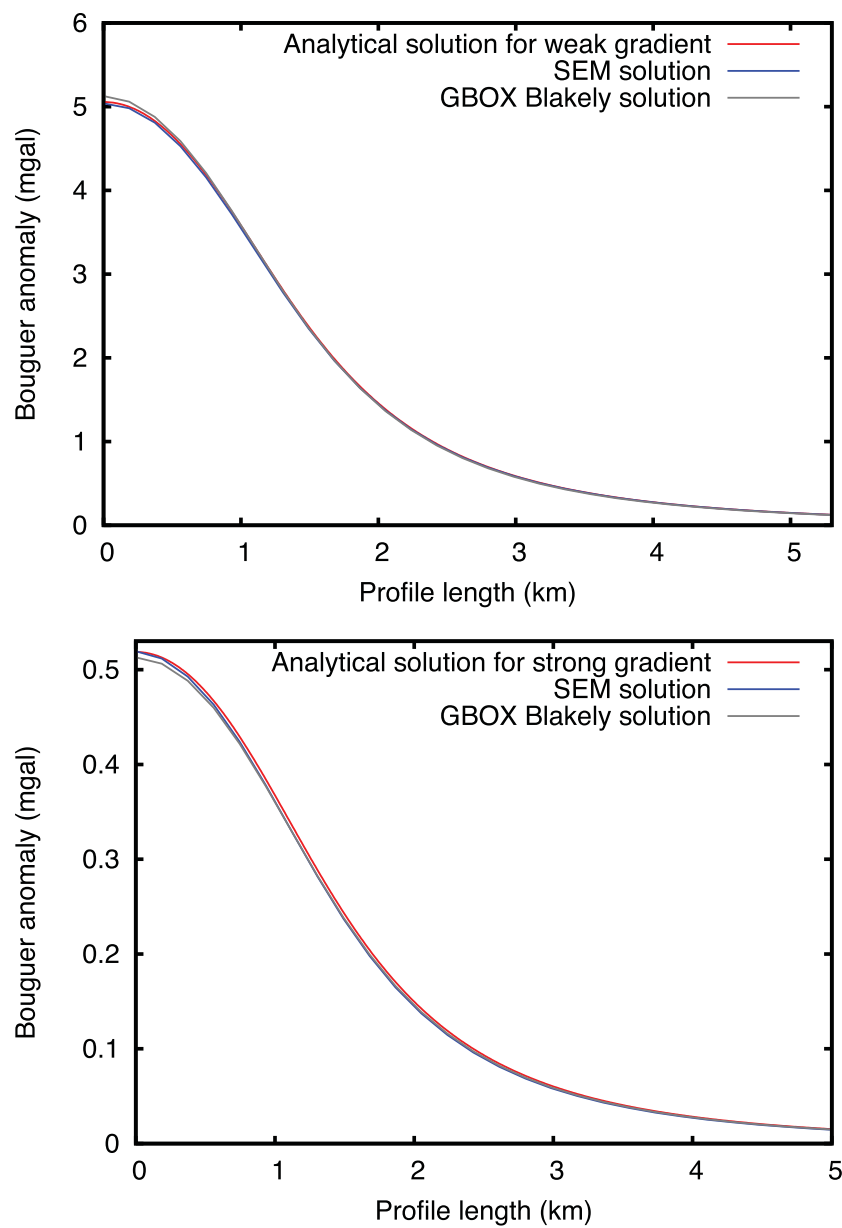

Figure 3. Comparison of gravity anomalies in the case of a density gradient inside a $2 \mathrm{~km} \times 1 \mathrm{~km} \times 1 \mathrm{~km}$ material block located at $1 \mathrm{~km}$ depth. A weak (top) and a stronger gradient (bottom) are tested. We get similar solutions when weak gradients are considered in both SEM and semi-analytical computations, but the SEM is slightly more accurate with a maximum error around 0.69 per cent just below the centre of the anomaly response. The Blakely approach gives errors up to around 1.5 per cent. In the case of a stronger gradient, the SEM gives a better result (error around 0.14 per cent) than the Blakely method (error around 1.1 per cent). In the SEM approach, five GLL collocation points are used along each spatial direction of a 3-D spectral element.

an error around 0.7 per cent. Furthermore, for a similar number of discretization points, the SEM takes $7 \mathrm{~s}$ to compute while GBOX takes $249 \mathrm{~s}$. The SEM technique is thus very promising for large scale computations and is much more competitive than the Blakeley technique. In all cases, equivalent or better accuracy and a drastically increased rapidity of the code is obtained for SEM when compared to GBOX.

\subsubsection{Density gradient in depth}

We now compute gravimetric anomalies produced by different density gradients from weak to high density variations in depth. Density anomalies $\Delta \rho$ are distributed in a prism $2 \mathrm{~km}$ long, $1 \mathrm{~km}$ wide and $1 \mathrm{~km}$ deep with its top edge located at $z_{t}=1 \mathrm{~km}$ depth:

$\Delta \rho=\frac{\Delta \rho_{o}^{3}}{\left[\Delta \rho_{o}-d\left(z-z_{t}\right)\right]^{2}}$,

where $d=0.001 \mathrm{~kg} \mathrm{~m}^{-4}$ is a test parameter given in Chakravarthi et al. (2002), and $z_{t}$ is the depth location of the top edge of the density anomaly. Two different values of $\Delta \rho_{o}$ equal to 100 and 1000 are tested. For $\Delta \rho_{o}=100$ a significant density gradient is obtained, and for $\Delta \rho_{o}=1000$ a weak gradient is obtained. A total number of GLL points in the SEM approach equal to 49 along the $z$-direction (depth) is introduced and corresponds to 12 spectral elements with an average spacing of $0.125 \mathrm{~km}$. We compare the SEM solution to that obtained with the Blakely semi-analytical computations performed with a 48-cell discretization along the $z$-direction. Both solutions are compared to the exact analytical solution of Chakravarthi et al. (2002). In Fig. 3, the SEM solutions are more accurate than semi-analytical solutions, with respective errors around 0.14 percent for the SEM and around 1.1 percent for the Blakely solution in the case of a value of $\Delta \rho_{o}$ $=100 \mathrm{~kg} \mathrm{~m}^{-3}$. If weak density gradients are considered, that is, for $\Delta \rho_{o}=1000 \mathrm{~kg} \mathrm{~m}^{-3}$, errors are approximately equal to 0.69 per cent for the SEM and 1.4 per cent for the Blakely semi-analytical solution. This means that the SEM is a better candidate to accurately model geological structures when density gradients are present, which is the case in almost all realistic cases. In addition, as above, SEM is much faster than GBOX by a similar factor of around 40 in terms of computational speed. 

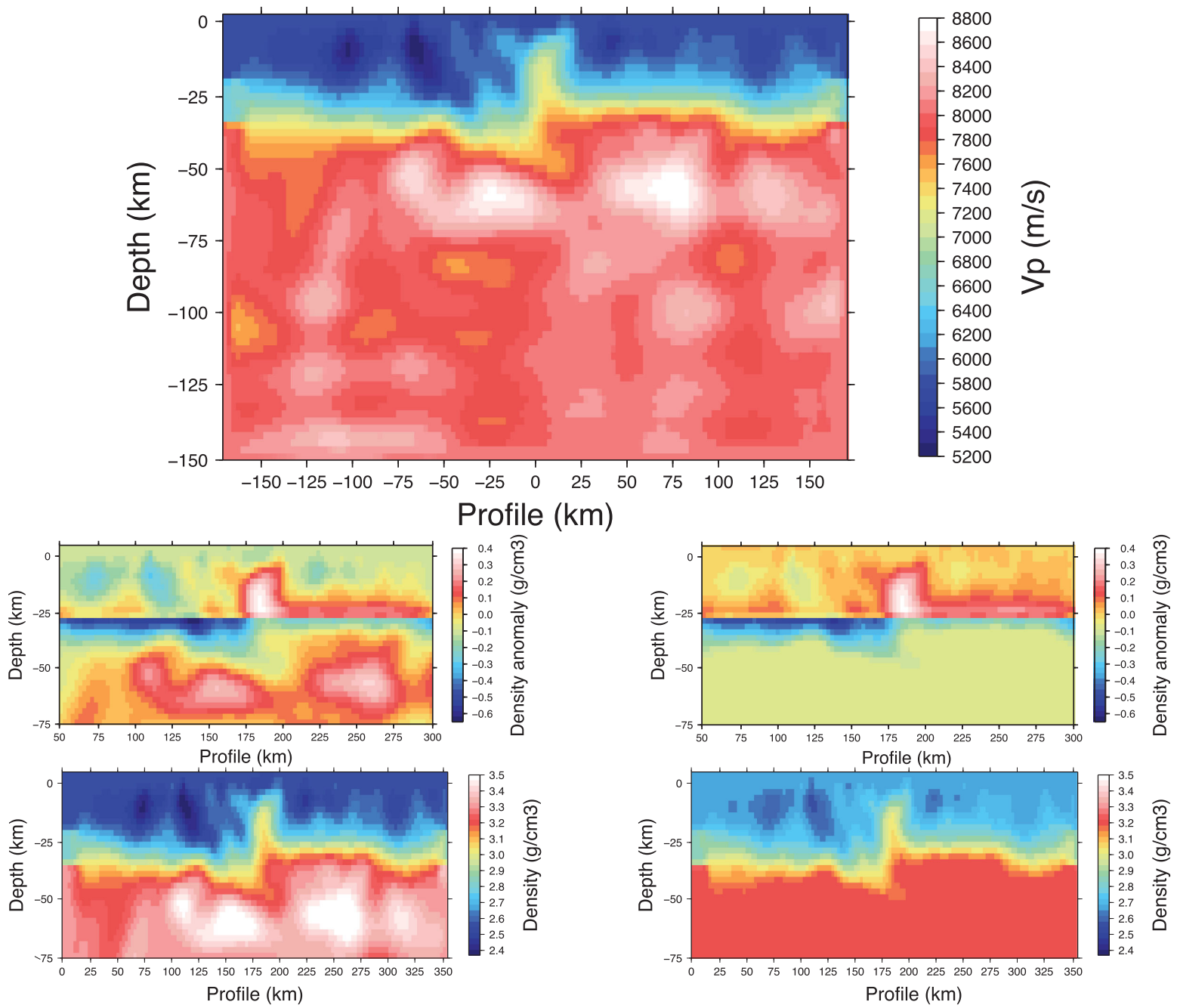

Figure 4. 2-D a priori seismic pressure velocity $V_{p}$ section (top), a priori density anomalies (middle) and total density distributions (bottom) using Birch (left) or Nafe-Drake (right) correlation laws.

\subsection{Western Pyrenees}

Let us now illustrate the accuracy of the SEM integration by computing the Bouguer anomaly for a 3-D density model of the western Pyrenees and comparing the results to the GBOX technique. The density model is derived by scaling a $V_{p}$ model (Wang et al. 2016) obtained by FWI of teleseismic $P$-waves (Monteiller et al. 2015) recorded by the western PYROPE profile (Chevrot et al. 2014). The 3-D density model is constructed by extracting a $V_{p}$ profile going from $\left(-2.6150^{\circ}, 42.071^{\circ}\right)$ to $\left(0.32167^{\circ}, 44.4323^{\circ}\right)$. To construct the density model we test two different scaling relations between $V_{p}$ and $\rho$ :

(1) A Birch linear scaling (Birch 1960, 1961) defined in the CGS unit system for $V_{p} \leq 7.4 \mathrm{~km} \mathrm{~s}^{-1}$ :

$\rho=\left(V_{p}+1.9\right) / 3$.

(2) A Nafe-Drake law (Ludwig et al. 1970; Brocher 2005) for $V_{p} \leq 7.4 \mathrm{~km} \mathrm{~s}^{-1}$ :

$\rho=1.6612 V_{p}-0.4721 V_{p}^{2}+0.0671 V_{p}^{3}-0.0043 V_{p}^{4}+0.000106 V_{p}^{5}$.

For these two scaling laws, we set $\rho=3.2 \mathrm{~g} \mathrm{~cm}^{-3}$ for $V_{p}>7.4 \mathrm{~km} \mathrm{~s}^{-1}$. We then set the density anomalies $\Delta \rho=\rho-2.67 \mathrm{~g} \mathrm{~cm}^{-3}$ in the crust (from the upper surface down to a Moho depth of reference located at $30 \mathrm{~km}$ ) and $\Delta \rho=\rho-3.27$ in the upper mantle (at depths larger than $30 \mathrm{~km}$ ). The dimensions of the 3-D computational grid are $340 \mathrm{~km} \times 150 \mathrm{~km} \times 20 \mathrm{~km}$, with a voxel discretization of $136 \times 61 \times 15$ points $(\simeq 2.5 \mathrm{~km} \times 2.5 \mathrm{~km} \times 1.5 \mathrm{~km}$ cell volumes $)$.

To avoid edge effects, the model is laterally extended over $50 \mathrm{~km}$ on each side of the mesh. Density values at the vertical edges are tapered to $2.67 \mathrm{~g} \mathrm{~cm}^{-3}$ in the top $30 \mathrm{~km}$ and to $3.2 \mathrm{~g} \mathrm{~cm}^{-3}$ below $30 \mathrm{~km}$ depth. Nafe-Drake scaled densities of the western profile are shown in Fig. 4. We then compute Bouguer anomalies along the profile using the semi-analytical and SEM techniques for both density distributions (Fig. 5). For a number of GLL points smaller than 4 in each cell, solutions are inaccurate, but for 5 points the agreement between the semi-analytical reference solution and the spectral-element computation is excellent. Consequently, in the following, a number of GLL points equal to 5 


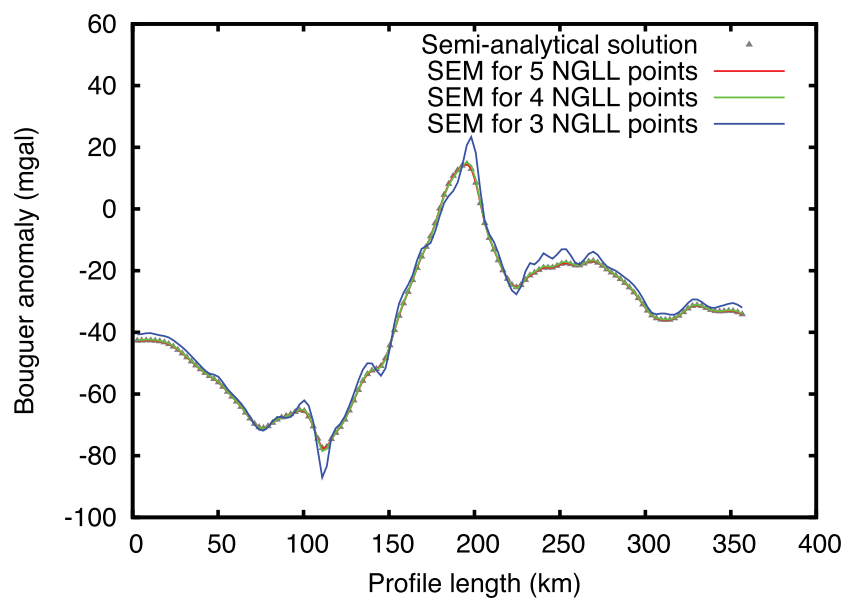

Figure 5. Comparison of gravity anomalies for an initial a priori model obtained with the Nafe-Drake relation (Fig. 4, bottom right), applying semi-analytical or spectral-element integration methods with different numbers of GLL ( $N G L L=3,4$ or 5) integration points along each spatial direction of a 3-D spectral element. The solutions are well superimposed. A spectral-element method of degree 4 with five GLL $(N G L L=$ degree +1$)$ collocation points along each direction is thus sufficient. Slight or strong shifts are respectively observed in the anomalies when using only three or four points instead of five.

is retained in all our spectral-element computations. In Fig. 6, we show the observed and computed anomalies with a priori models built based on Birch or Nafe-Drake scaling relations applied to the $V_{p}$ model. A strong positive jump of around 90 mgal is observed north of the Pyrenees, in the Mauléon basin. The a priori density model derived from the Nafe-Drake law provides a much better fit to the gravity data. In the remainder of this paper, we will therefore use only the Nafe-Drake scaled density model as our a priori model in all the inversions.

\section{GRAVITY DATA SET}

We have extracted a data set of Bouguer gravity anomalies measured on the ground surface from the Bureau Gravimétrique International (BGI) database (Fig. 7). For the inversions we inverted the data interpolated on the western PYROPE profile passing through the Mauléon basin. The $V_{p}$ model is taken from the teleseismic FWI of Wang et al. (2016) on a SEM grid that includes the profile. This $V_{p}$ model is scaled to density using the Nafe-Drake relation (16). To construct the mesh, we take into account the Earth curvature from the WGS84 reference ellipsoid, and use the ETOPO1 model for topography (Amante \& Eakins 2009). To model the oceans, between the sea floor and the ellipsoid level densities are set to a constant value of $1030 \mathrm{~kg} \mathrm{~m}^{-3}$. To avoid edge effects, density values are again tapered over an extended outer domain of $50 \mathrm{~km}$ surrounding the profile, to reach the reference values 2670 and $3270 \mathrm{~kg} \mathrm{~m}^{-3}$ respectively at depth $z<30 \mathrm{~km}$ and at depth $z>30 \mathrm{~km}$. The Bouguer anomaly profile predicted by our a priori density model is shown in Fig. 8 . The computed anomalies are close to the ground observations, but significant deviations are observed in the Ebro and Aquitaine basins. Besides, the calculations along the profile are not significantly impacted by density anomalies at distances larger than $50 \mathrm{~km}$ from the profile. For the inversions we will thus perform the calculations in a $40 \mathrm{~km}$-wide slice containing the longitudinal seismic profile. We also notice that gravity anomalies computed on a spherical Earth or on the WGS84 ellipsoid differ by less than 1 mgal. However, the surface topography has a significant effect in the anomaly computations. In Fig. 8 (bottom), we show the difference between simple and complete measured Bouguer anomalies. One can see that the topography corrections reach values as high as $7 \mathrm{mgal}$ across the Pyrenees and $3 \mathrm{mgal}$ in northern Spain, and are much less significant in the Aquitaine basin. The effect of topography is thus not negligible. The difference between the computed and measured complete Bouguer anomalies varies from around -19 to $14 \mathrm{mgal}$ due to mass defects in the Pyrenees and excesses of mass in the Aquitaine basin, where lower densities should exist due to the presence of thick sedimentary layers close to the surface.

\section{INVERSION METHOD}

\subsection{Inversion procedure}

Some of the main problems encountered when inverting gravity data are the choice of the a priori model, the density of observations (data coverage) along and around the profile, and the way the topography is taken into account. FWI mainly constrains the velocity model beneath the transect due to the fact that the seismic array is dense along the profile, with around $5 \mathrm{~km}$ spacing between stations, but is sparse over distances of 20 to $40 \mathrm{~km}$ apart from the profile. Consequently we will perform data inversion only in a $40 \mathrm{~km}$ wide domain.

The inversion consists in searching for the model that minimizes the misfit function:

$\chi(\Delta \rho)=\frac{1}{2}\left\|\Delta g_{\text {obs }}-\Delta g(\Delta \rho)\right\|_{l^{2}}^{2}+\frac{1}{2} \lambda\left\|\Delta \rho-\Delta \rho_{o}\right\|_{L^{2}}$, 


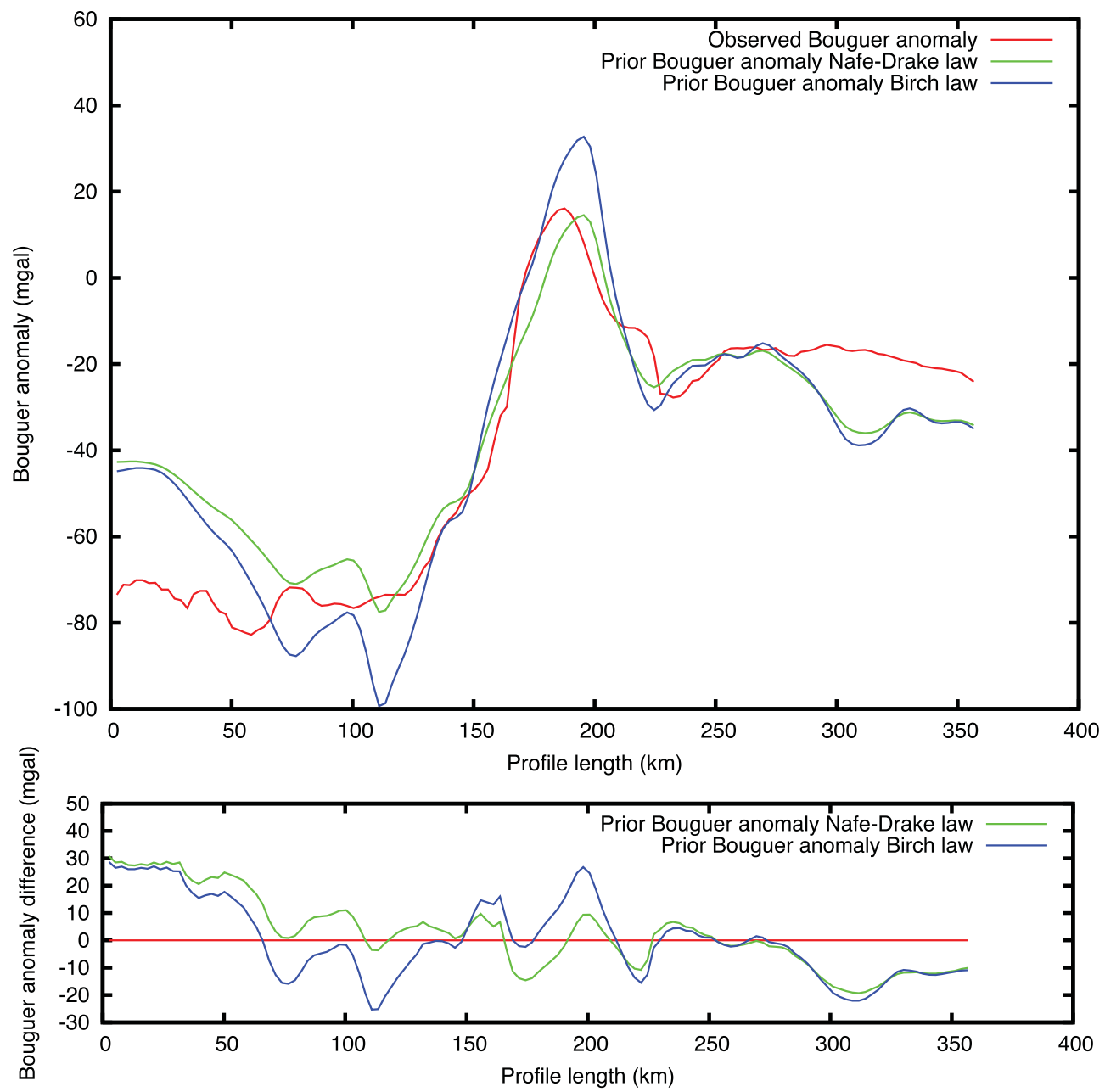

Figure 6. Top: gravity anomalies predicted by the density models obtained with the Birch and Nafe-Drake scaling relations. The Nafe-Drake relation gives results in closer agreement to the observed data. Bottom: differences between computed and observed anomalies. The smallest misfit is obtained with the Nafe-Drake relation.

where $\Delta \rho_{o}$ is the a priori density anomaly model, $\Delta \rho$ is the density anomaly to be retrieved after inversion, $\Delta g_{\text {obs }}$ the observed gravity anomaly, and $\lambda$ the damping parameter that regularizes the inverse problem. Let us denote $m$ the model vector containing the density anomalies $\Delta \rho$. This model vector is obtained by solving the linear system of equation:

$\left[\begin{array}{c}S \\ \lambda I_{d}\end{array}\right] m=\left[\begin{array}{c}\Delta g_{\mathrm{obs}} \\ \lambda m_{o}\end{array}\right]$,

where $I_{d}$ is the identity matrix and $m_{o}=\Delta \rho_{o}$ is the a priori model. The solution of eq. (18) is obtained by an iterative LSQR algorithm (Paige \& Saunders 1982). At each $i$ th LSQR inversion cycle we solve a linearized version of the system of eq. (18) as follows:

For $1 \leq i \leq$ iter

$$
\begin{array}{rlrl}
{\left[\begin{array}{c}
S \\
\lambda I_{d}
\end{array}\right]\left[\Delta m^{i}\right]} & = & & {\left[\begin{array}{c}
\Delta g_{\mathrm{obs}}-\Delta g\left(m^{i-1}\right) \\
-\lambda\left(m^{i-1}-m_{o}\right)
\end{array}\right]} \\
m^{i} & = & m^{i-1}+\Delta m^{i}
\end{array}
$$

Every 3 or 4 cycles: the model $m^{i}$

is tapered towards a reference model $m_{o}$

in the lateral boundary extents

End for,

where $\Delta m^{i}$ is the perturbation of the density anomaly model $\Delta \rho$ at the $i$ th LSQR cycle, and $\Delta g_{\text {obs }}$ and $\Delta g$ are respectively the observed and calculated data for the current model. At each new LSQR cycle the model in the right-hand side $m^{i}$ is replaced with the solution computed at the previous inversion cycle. Every three or four cycles, a tapering of the current model $m^{i}$ to the reference model $m_{o}$ is applied from the 


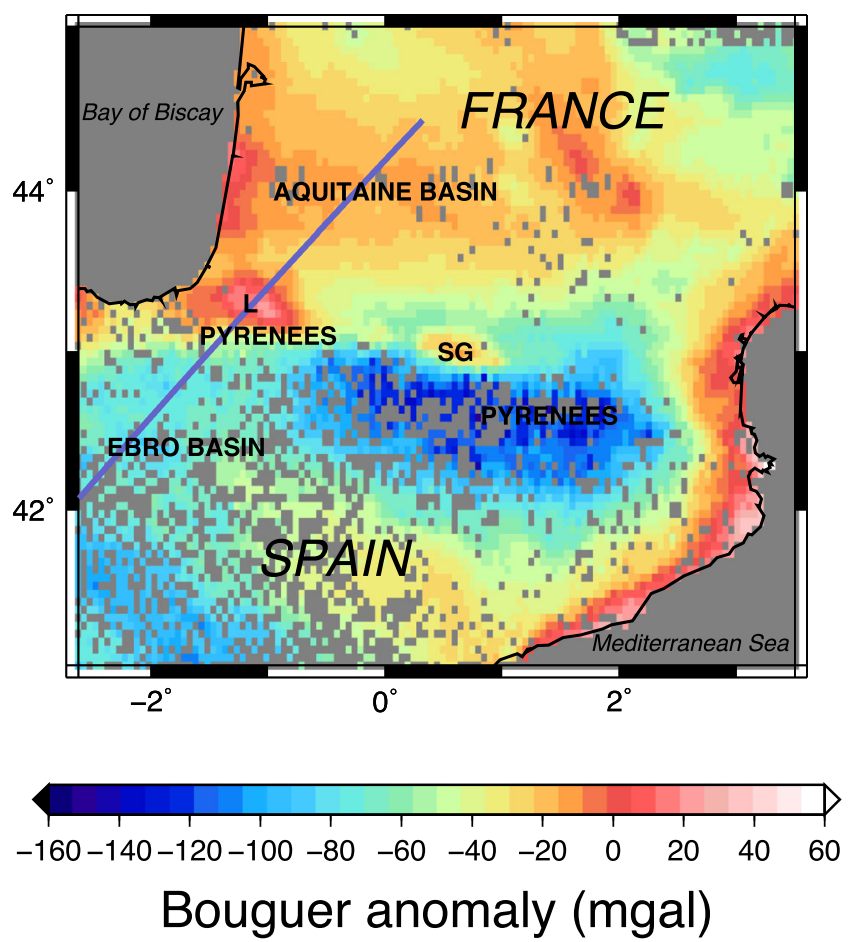

Figure 7. Complete Bouguer gravity anomaly data measured on the ground and distributed in space in the Pyrenees region (extracted from the worldwide BGI/Bureau Gravimétrique International database). The seismic profile along which we invert seismic and gravity data is depicted by the blue line. The dots represent the locations where gravity measurements are missing. L and SG denote respectively the Labourd and Saint-Gaudens locations showing positive Bouguer gravity anomalies.

inner boundary of the computational domain to the outer boundary through the $50 \mathrm{~km}$ lateral extents in order to damp possible edge effects. However, this linear system is not well conditioned because the sensitivity matrix decreases as a function of the square of the distance to the observation point. We thus introduce a diagonal preconditioning operator $H$ defined as (Li \& Oldenburg 1998; Pilkington \& Hildebrand 2000; Li \& Oldenburg 2003):

$H=\left[\begin{array}{ccccc}z_{1} & & & \\ & & & \\ & \cdots & & \\ & & z_{i} & \\ & & & \\ & & & \\ & & & z_{n}\end{array}\right]$,

where $z$ is the vertical distance from the observed data location to the density anomaly $m$ location. At the $i$ th LSQR process cycle the new set of equations reads:

$$
\begin{aligned}
& \begin{array}{lll}
\bar{S} & = & S H
\end{array} \\
& \bar{m}^{o} \quad=\quad H^{-1} m^{o} \\
& {\left[\begin{array}{c}
\bar{S} \\
\lambda I_{d}
\end{array}\right]\left[\Delta \bar{m}^{i}\right] \quad=\quad\left[\begin{array}{c}
\Delta g_{\mathrm{obs}}-\Delta g\left(m^{i-1}\right) \\
-\lambda\left(m^{i-1}-m_{o}\right)
\end{array}\right]} \\
& \begin{array}{lll}
\bar{m}^{i} & = & \bar{m}^{i-1}+\Delta \bar{m}^{i} \\
m^{i} & = & H \bar{m}^{i}
\end{array}
\end{aligned}
$$

Every 3 or 4 cycles: the model $m^{i}$

is tapered towards a reference model $m_{o}$

in the lateral boundary extents

End for,

where the depth-normalized model is $\bar{m}^{i}=H^{-1} m^{i}$. In order to accelerate the computations and to be able to handle large model sizes in the future, we perform the inversions in a wavelet-compressed model space, following an idea that has been applied to magnetic data 

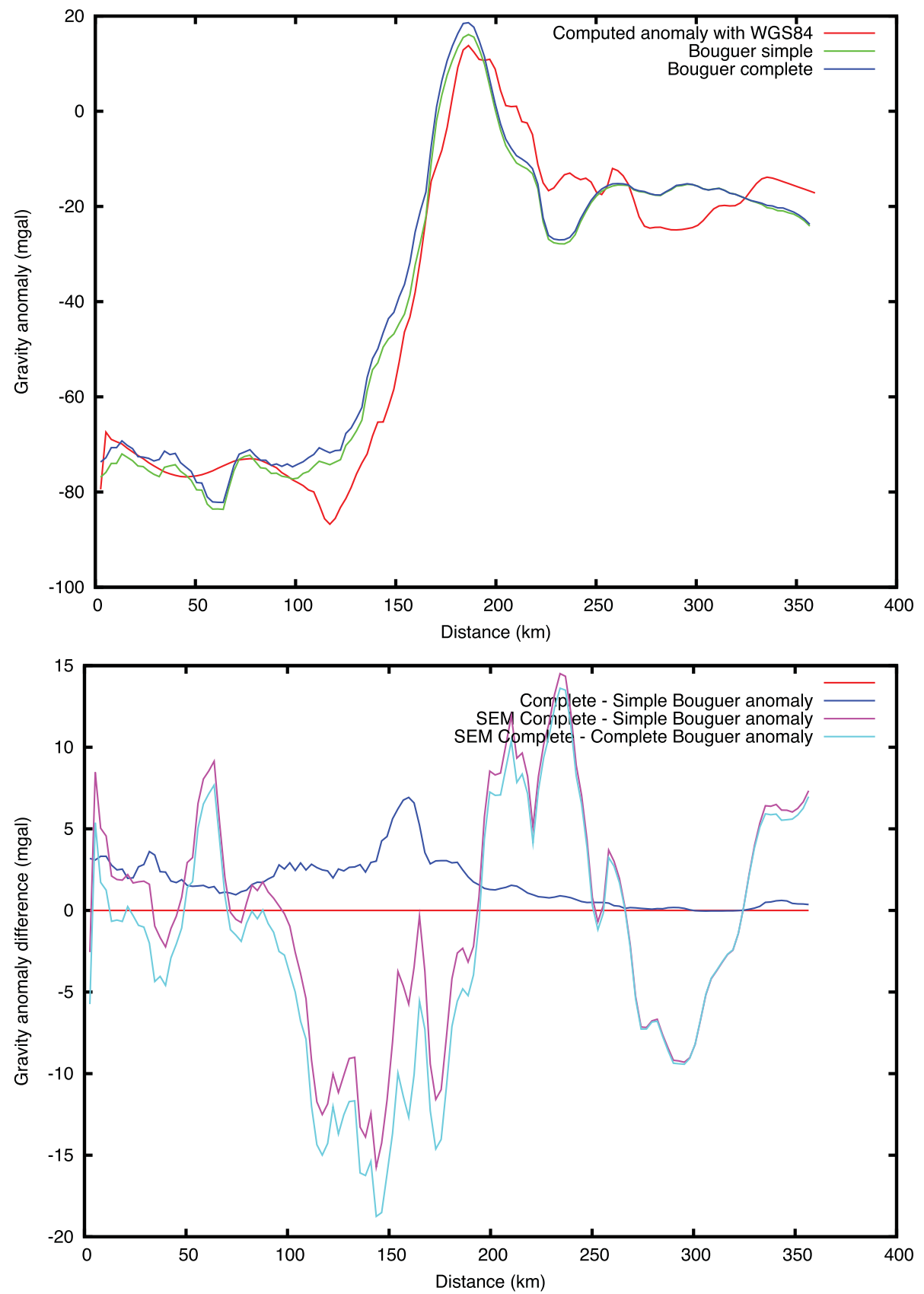

Figure 8. Top: a priori complete Bouguer anomalies ('WGS84 red solid line') computed with the SEM for a full 3-D density model including ETOPO1 topography, Atlantic Ocean (sea level) and the WGS84 Earth reference ellipsoid. The simple (green curve) and complete (blue curve) Bouguer anomalies are measured ground data that are interpolated on the 1-D profile and compared to the Bouguer gravity anomaly computed with the SEM. A small shift is observed between the two anomaly curves. Bottom: difference between complete and simple Bouguer anomalies. One can observe that positive differences are mainly concentrated in the Pyrenees (up to $7 \mathrm{mgal}$ ) and the Spanish regions (up to around $3 \mathrm{mgal}$ ) due to significant topography effects, and are much less pronounced in the Aquitaine basin, as expected. One can also notice that topography correction effects are significant.

inversion by Li \& Oldenburg (2003). We use the same wavelet compression procedure as the one applied to sensitivity kernels in seismic tomography (Chevrot et al. 2012) or in the TOMOFAST3D code (Martin et al. 2013). The reader is referred to these articles for more details. By applying a (CDF4) fourth-order Cohen-Daubechies-Fauveau (Cohen et al. 1992) lifting scheme to the sensitivity matrix in each direction of the 3-D space, the matrix can be compressed by a factor of about 80 per cent for an error of less than 1 per cent in the evaluation of the response. We apply the compression wavelet operator three times, once along each spatial dimension. If one compresses too much, too much information is lost and the inversion process is not efficient due to the fact that many parts of the domain can have an infinity of solutions, since the compressed sensitivity matrix has elements equal to zero there. A sparse sensitivity kernel is obtained after thresholding the wavelet coefficients of the wavelet transform matrix; this is why we say that the sensitivity kernel is compressed. This threshold is defined once and for all before the whole inversion process by keeping wavelet coefficients of the transformed matrix that have values lower than $10^{-3}$ of the 

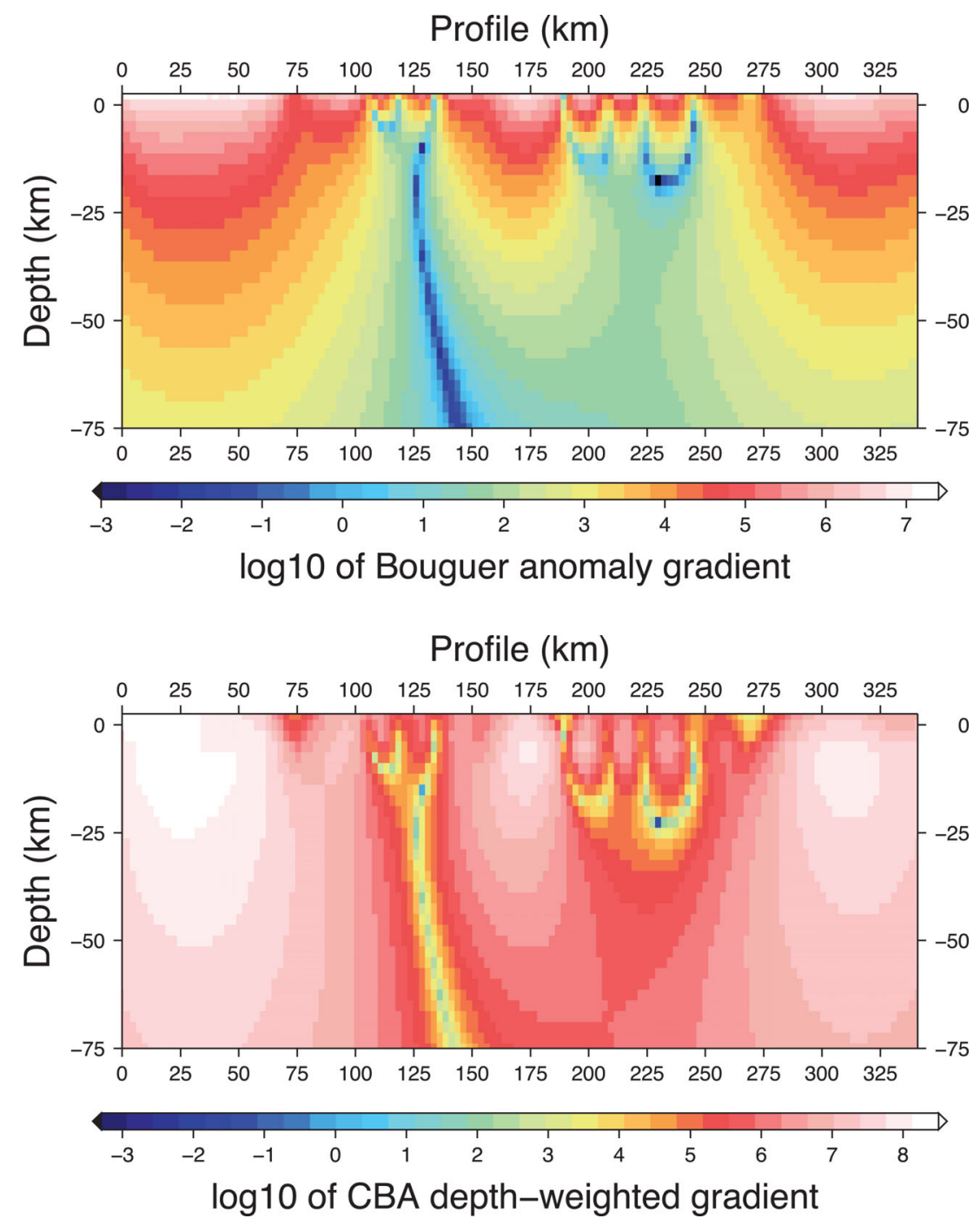

Figure 9. Top: gradient of the complete Bouguer anomaly (CBA) misfit function for the Nafe-Drake scaled a priori density anomaly model. The sensitivity to density perturbations is mainly concentrated in the first $20 \mathrm{~km}$ depths. Bottom: depth-preconditioned gradient of the CBA data misfit for the a priori density anomaly that is normalized linearly with depth. The gradient is increased with depth and sensitivity is thus enhanced at depth compared to the non-depth-weighted gradients. The depth-weighted sensitivity is increased at depth, and then density is more perturbed at depth during the inversion process.

maximum wavelet coefficient value. The following linear system is then solved in the compressed space:

$$
\begin{aligned}
S^{c} & & & \bar{S}^{c} \\
m^{c} & & & \\
& \text { For } 1 & \leq i \leq \text { iter } & \\
{\left[\begin{array}{c}
S^{c} \\
\lambda I_{d}
\end{array}\right]\left[\Delta m^{c}\right] } & & & \\
\Delta m & & & \\
m & & & {\left[\begin{array}{c}
\Delta g_{\text {obs }}-S^{c}\left(m^{c}\right) \\
-\lambda\left(m^{c}-m_{o}^{c}\right)
\end{array}\right] } \\
m & & & w^{T} \Delta m^{c} \\
m^{c} & & & m+\Delta m \\
& & & w m
\end{aligned}
$$

Every 3 or 4 cycles: the model $m$

is tapered towards a reference model $m_{o}$ in

the lateral boundary extents

End for, 

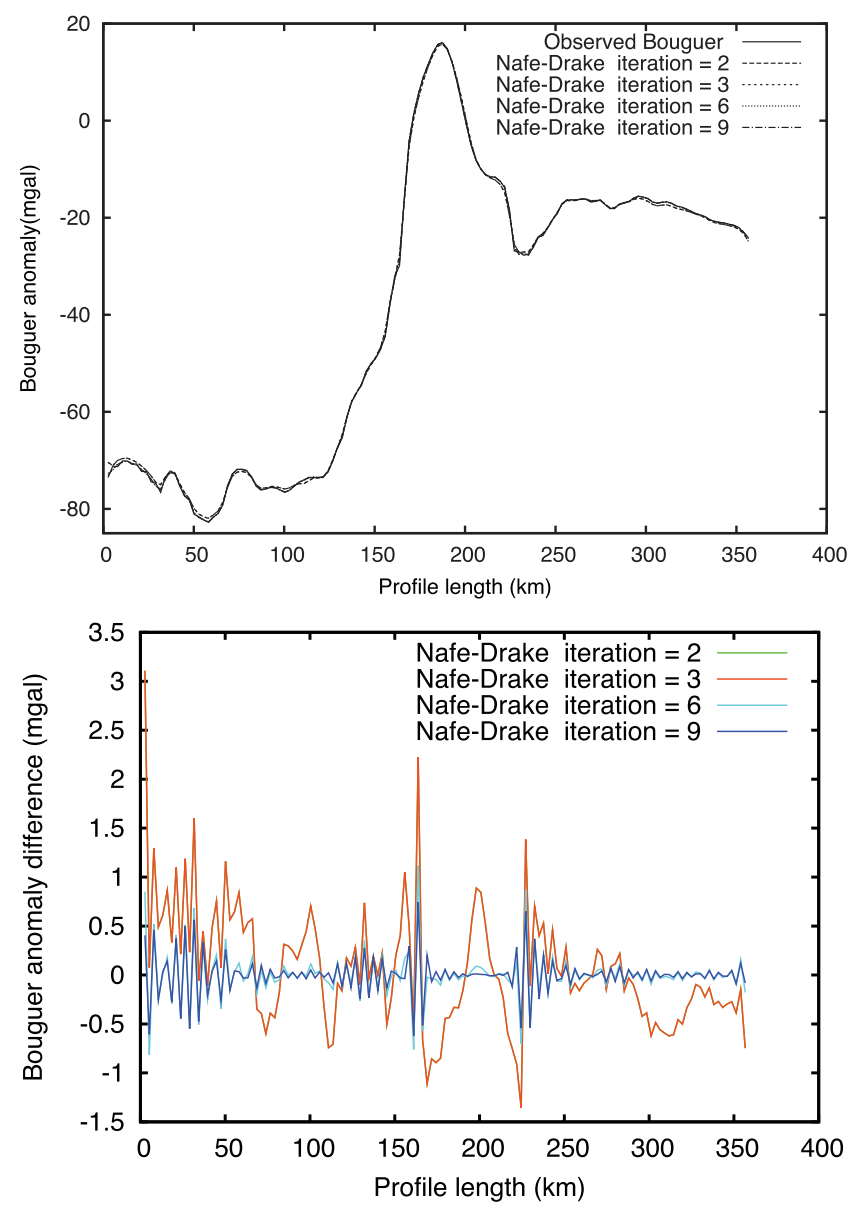

Figure 10. Top: observed BGI anomalies and computed anomalies after different inversions and different a priori model reinitializations, when using initial Nafe-Drake law-based density anomalies of Fig. 1. A regularization parameter $\lambda=20$ has been used. One can observe that the solutions are very well superimposed, even if the models obtained after inversion are different in depth. After six and even nine global iterations of the entire inversion process the Nafe-Drake correlation-based models have converged, with much smaller gravity residuals than after three global iterations. One global iteration means one LSQR inversion process. It is interesting to note that even if the problem is linear it is crucial to update the a priori model several times in order to converge. Bottom: the impact of the number of inversion cycles on the decrease of the gravity residuals is shown when we plot the differences between the computed and observed BGI anomalies after several inversions. The misfit is drastically reduced further after six and even nine inversions.

where $w^{T}$ is the inverse wavelet operator applied to the compressed model in order to reconstruct the model $m$ in the physical (uncompressed) space.

\subsection{Gradient of the misfit function}

The gradient of the misfit function (17), without considering the depth weighting pre-conditioner, is defined by:

$\nabla \chi(m)=S^{T}\left[\Delta g_{\mathrm{obs}}-\Delta g(m)\right]+\lambda\left(m-m_{o}\right)$,

where $m=\Delta \rho$ and $m_{o}=\Delta \rho_{o}$ are respectively the inverted and a priori density anomaly models. Physically, this gradient gives the direction along which one needs to perturb the density to make the misfit function decrease. Fig. 9 shows the gradient computed in the a priori model. The gradient decreases by almost three to four orders of magnitude over $20 \mathrm{~km}$ along the depth dimension. This means that the data sensitivity to model perturbations is not really significant at depths larger than approximately $15-20 \mathrm{~km}$. This gives a first indication that large-scale structures are already well captured by the FWI model and cannot be significantly improved by the gravity data inversion. On the other hand, small-scale shallow structures are required to improve the fit to gravity anomalies.

If a depth-weighting pre-conditioner is introduced, the gradient of the misfit function (17) then reads

$\nabla \chi\left(m^{\prime}\right)=H^{-1 T} S^{T}\left[\Delta g_{\mathrm{obs}}-\Delta g(m)\right]+\lambda\left(m^{\prime}-m_{o}^{\prime}\right)$, 

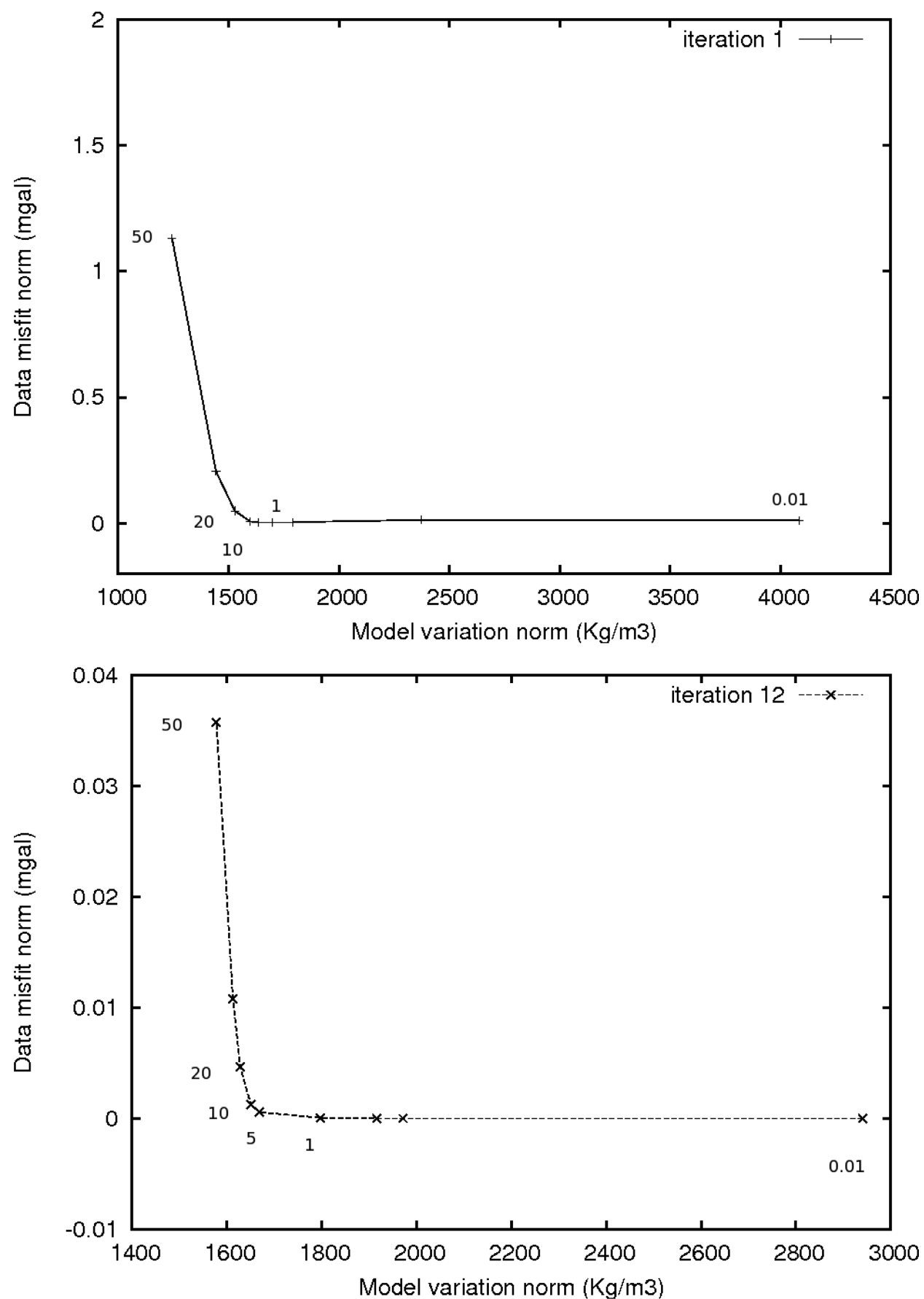

Figure 11. Top: L-curve for the first iteration of the CBA data inversion and different $\lambda$ regularization parameters $0.01,0.1,0.5,1,5,10,20,30,50$ and 100 . An apparent inflection point appears in a region where data misfit reaches a reasonably low value. The inflection of the L-curve appears for damping parameters $\lambda$ ranging between 5 and 10 . For a value of 10 we get a misfit norm greatly reduced compared to the case of a high damping value of 50 . Bottom: That $L$-curve is shown after the final iteration of the inversion process. One can see that an inflection region also appears for damping values between 5 and 10 , and misfits are even more reduced compared to the solution after the first iteration. Between the first and last inversion cycle the misfit decreases as expected in each case by around two orders of magnitude. It is thus important to perform more than one global inversion cycle to reduce the misfit.

where $m^{\prime}=H^{-1} \Delta \rho$ and $m_{o}^{\prime}=H^{-1} \Delta \rho_{o}$ are respectively the inverted and a priori density anomaly depth-weighted models. In Fig. 9 the depth-weighted misfit gradient is enhanced at depth and thus the perturbations of the model are more pronounced at depth than close to the surface, even for depths larger than $20 \mathrm{~km}$.

\section{NUMERICAL RESULTS OF THE INVERSION}

An appropriate choice for the regularization parameter $\lambda$ has to be made in order to numerically stabilize the inversion process and obtain models that provide a good fit to the data. To determine a suitable $\lambda$ value to use, we have thus performed inversions with $\lambda$ values varying 

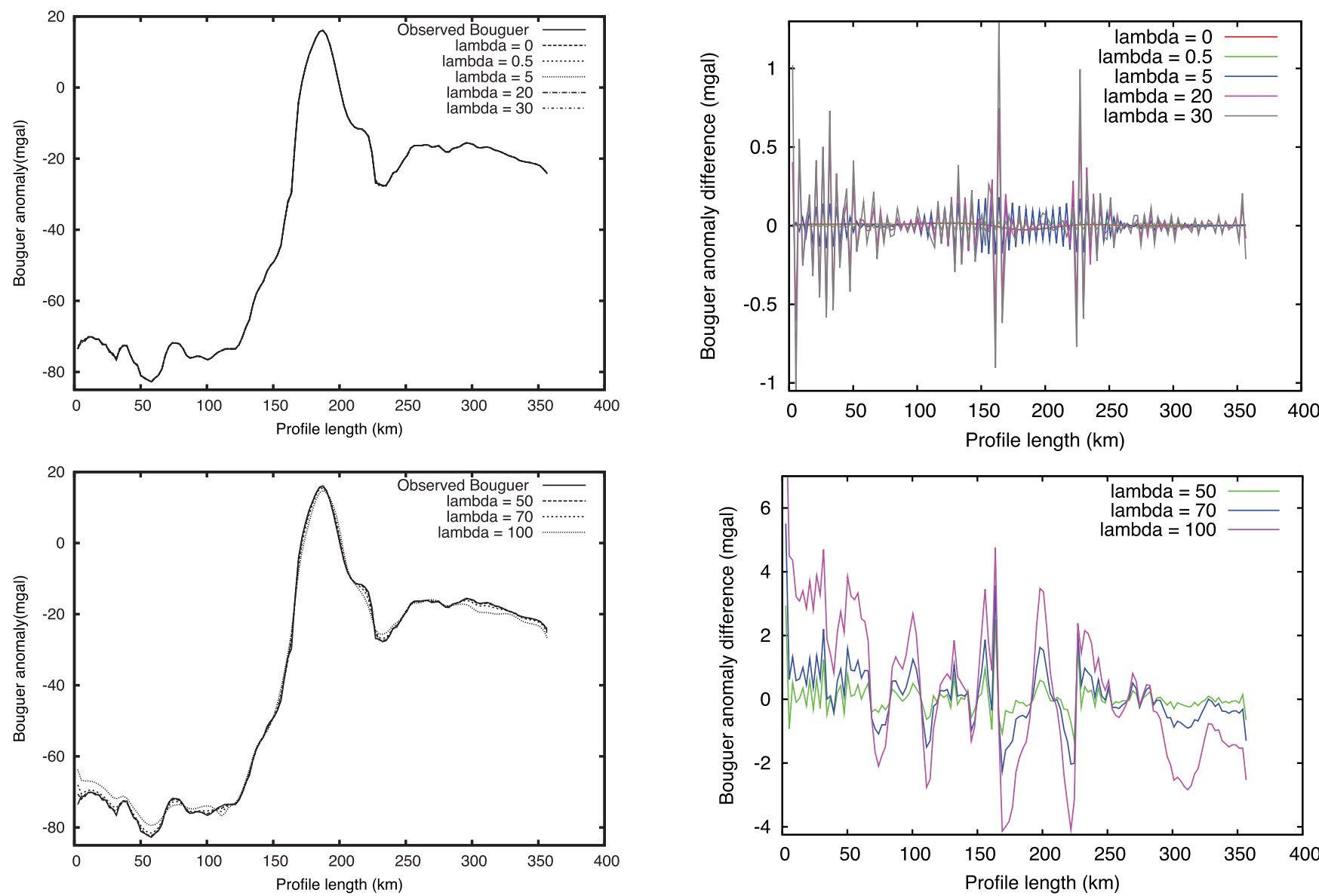

Figure 12. Inverted complete Bouguer gravity anomaly profile (left) and residual errors (right) obtained after inversion with different regularization parameters $\lambda$ varying from 0 to 100 . Increasing the regularization drastically reduces the spurious modes near the surface in both the models and the computed gravity data. One can see that for $\lambda<30$ the response is well reconstructed, while for $\lambda>30$ the response exhibits some discrepancies and has not really converged towards the measured gravity data.

from 0.01 to 50. Usually the LSQR algorithm converges after 300 iterations for each $i$ th LSQR cycle. Furthermore, three global cycles of the inversion problem including four inner loops at each cycle have been used to converge efficiently towards the solution. As explained in the previous section, at each inner loop iteration $i$ of a given global cycle, the new model obtained at the end of the previous iteration $i-1$ is introduced as an a priori model for the current inversion $i$. In Fig. 10, the decrease of the misfit between observed and computed data is shown for the different inversion cycles and for a damping coefficient $\lambda=20$. Solutions have almost converged after three inversion cycles but the residuals are even more reduced after six or even nine inversion cycles. In some tests, not shown here by sake of brevity, the misfit after two inversion cycles is much lower for the Nafe-Drake a priori model than for a Birch a priori model. The Birch correlation-based model has not totally converged even after nine iterations and this is another reason why inversion results are not shown here for Birch-scaled models.

For values $\lambda<10$, inverted density models exhibit spurious artefacts close to the surface. For $\lambda<1$, solutions are non-physical. For $\lambda>20$, artefacts in the solutions have much smaller amplitudes, and for even larger values of $\lambda$ the solutions are getting closer to the prior model due to strong damping. Fig. 11 shows the data misfit as a function of the $L^{2}$ norm of the density model. These curves have a typical $L$-curve shape (Hansen 2001), with an inflection point for $\lambda$ values between 5 and 20. In this range, the inverted models provide a good trade-off between reasonably low data misfit and a reasonable norm of density perturbations that are sufficiently important to get away from the a priori model without introducing numerical artefacts. In Fig. 11 (top), at the first iteration the inflection point corresponds to a $\lambda$ value around 20 but at the last iteration, which is iteration 12 (Fig. 11-bottom) the inflection point occurs for a $\lambda$ value around 10 that gives a smaller misfit. We therefore retain a value $\lambda=10$ in the inversion. One should note that, between the first and the last cycle of the inversion process, the misfit has decreased by at least one or two orders of magnitude.

In Fig. 12, the gravity response computed after inversion with different damping parameters varying from 0 to 100 is compared to the real data measured along the seismic profile. Increasing the regularization drastically reduces the spurious modes near the surface in both the models and their related gravity responses obtained after inversion. For values of $\lambda$ between 0 and 30, gravity anomalies are almost superimposed to the measured gravity anomaly, while for larger values $(50,70$ and 100) convergence of the inversion process is not totally 

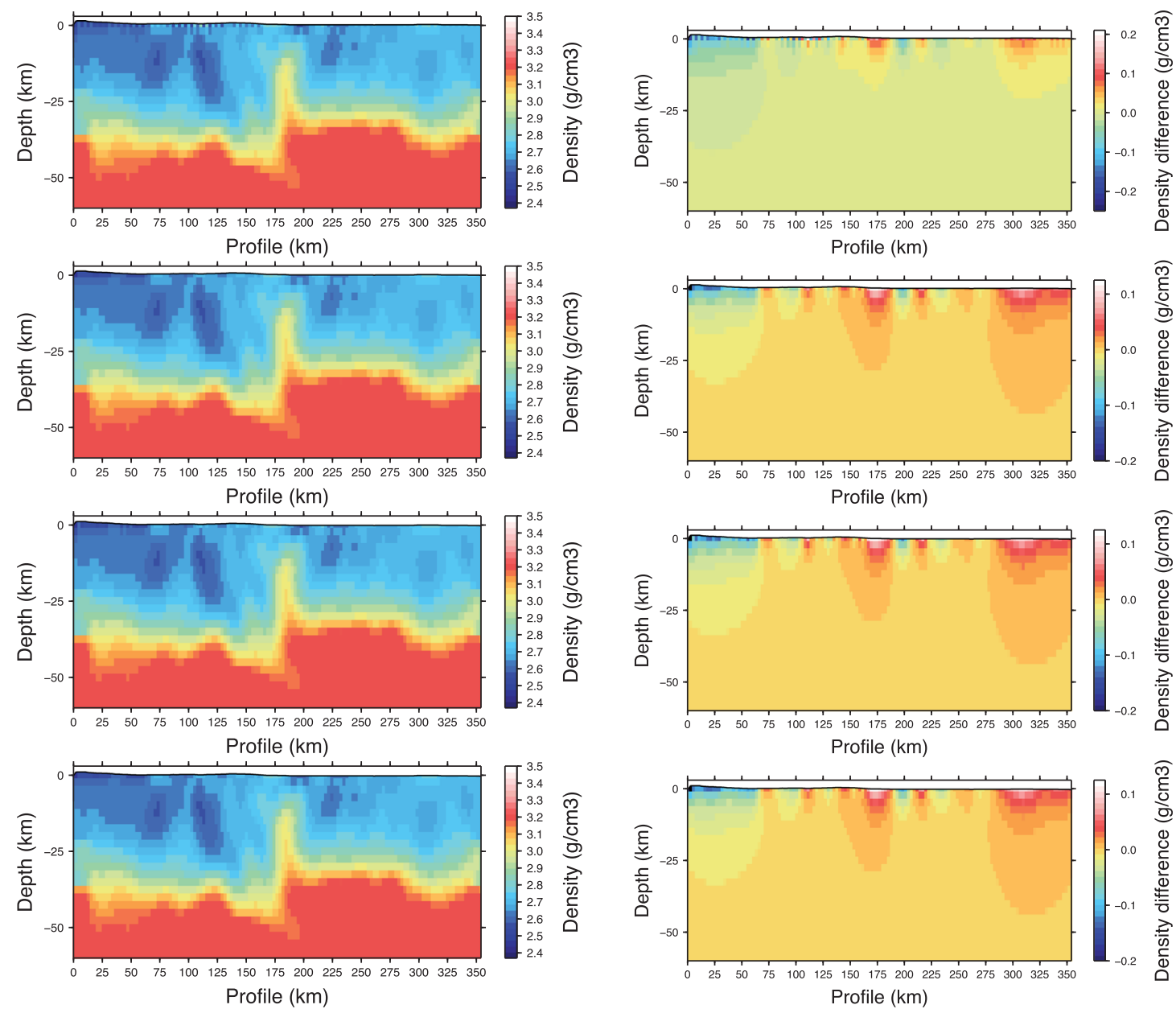

Figure 13. Total density (left) and density perturbation (right) with respect to the a priori model obtained after the three cycles of the inversion process, using different regularization parameters $\lambda$ (from top to bottom: $\lambda=1,5,20$ and 50).

achieved. However, low $\lambda$ regularization values produce noisy density models. Therefore, a range of damping parameters between 5 and 20 seems optimal to produce acceptable solutions according to the L-curves of Fig. 11. In Fig. 13, different $\lambda$ damping values are tested. The related inverted density models and differences between inverted models and a priori models are shown. For damping values higher than 50 , the smoothing seems to be too high and the model cannot be further improved and rather remains around the given a priori model. For values between 1 and 30, solutions seem to evolve, while for damping values lower than 5, for instance equal to 1 (top figures), spurious modes appear close to the surface. For damping values lower than 0.1 , solutions do not seem to make sense from a physical point of view. For values larger than 5 the spurious modes disappear. This is consistent with the range of $\lambda$ values around the apparent inflection point of the $L$-curve. Besides, the density model is almost the same in the whole domain except close to the surface in the southern (Spanish) part of the profile in the Ebro basin. In the inflection part of the $L$-curve, values around $\lambda=10$ lead to densities lower than $2550 \mathrm{~kg} \mathrm{~m}^{-3}$ over approximately 7-10 km depth that can be interpreted as basin density values. Full joint seismic and density inversions should thus be performed at higher frequencies to better image the basin density.

We have also performed some tests without constraint (using a tiny $\lambda$ value of $10^{-30}$ ). One can observe in Fig. 14 that the model is then stretched in depth and that solutions have large local spatial variations, with significant artefacts close to the surface, in the first $10 \mathrm{~km}$ and even almost everywhere in the model; in addition, significant changes are present even below the mantle-crust interface. Furthermore low density values are not present close to the topography, which is not consistent with observed Aquitaine basin densities. Low density values should be expected for sedimentary basins like the Aquitaine or Ebro basins (density should typically range between 2200 and $2550 \mathrm{~kg} \mathrm{~m}^{-3}$ ). Indeed, density models obtained in this area using compaction laws and correlations with well-log density samples show variations from 1700 to $2500 \mathrm{~kg} \mathrm{~m}^{-3}$ in the first $5 \mathrm{~km}$ (Grandjean 1994), while the a priori Nafe-Drake scaled densities are generally overestimated at shallow depths as noted by Brocher (2005). In our inversions we are able to define values that are more consistent with the density values modelled or observed close to the surface. 

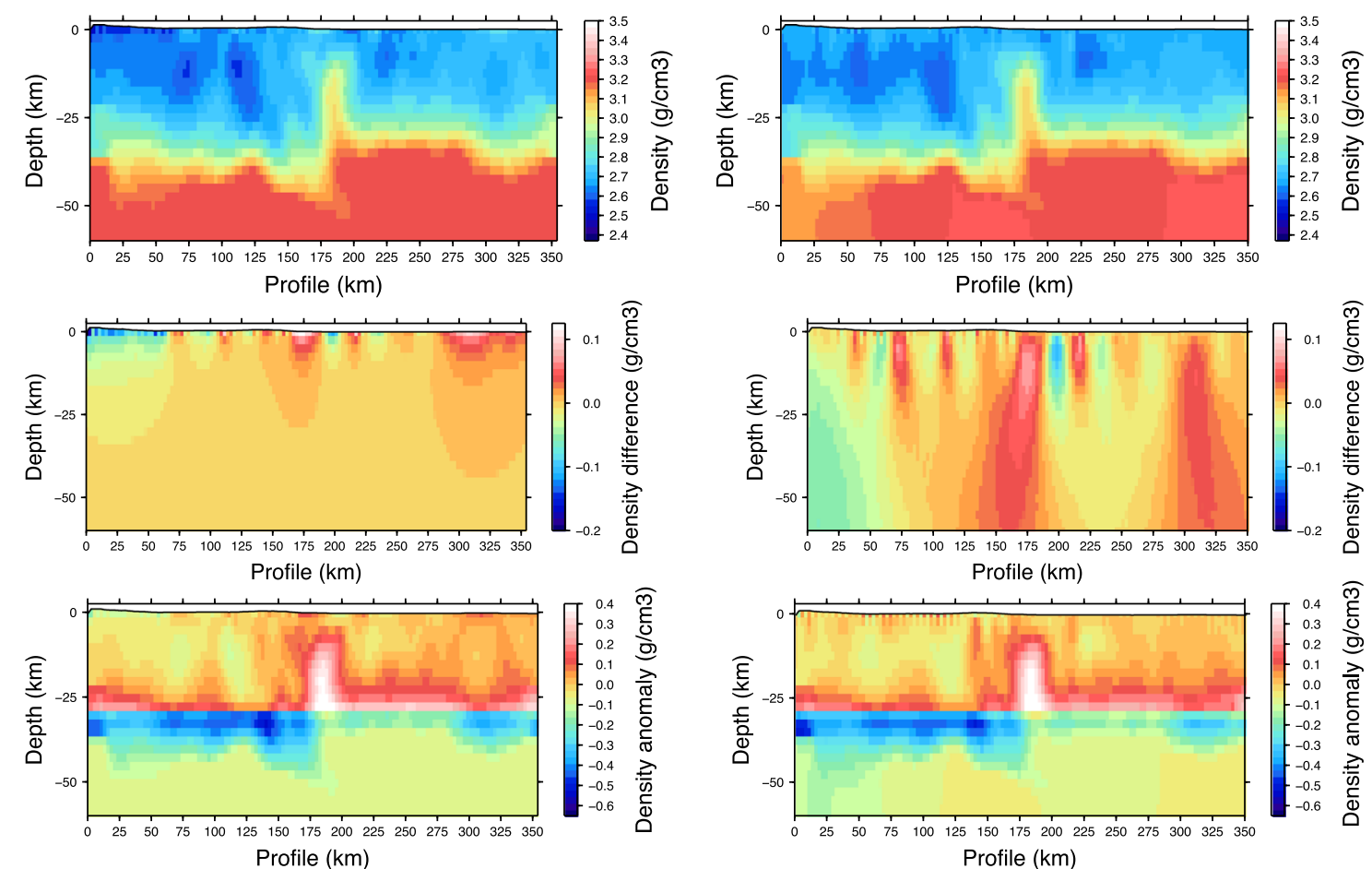

Figure 14. From top to bottom, density distributions after inversion, differences between inverted and a priori total densities, and density anomalies are shown for (left) an optimal regularization parameter $\lambda$ of 10 and (right) for almost no constraints in the misfit function (very small $\lambda \ll 1$ ). For $\lambda=10$, densities are almost the same in the whole computational domain except close to the surface in the first $7-10 \mathrm{~km}$ depth in the southern part of the Pyrenees. These low density structures close to the surface seem to correspond to sedimentary basin layers. However, for small $\lambda$ values, solutions do not seem to be physical since they exhibit strong numerical artefacts that appear almost everywhere as well as low densities close to the surface that are not consistent with expected Aquitaine basin densities.

\section{CONCLUSIONS AND FUTURE WORK}

We have shown gravity modelling results in 3-D realistic cases using a new numerical approach based on a high-order polynomial integration of each density volume contribution to the Bouguer gravity anomalies. Four to five GLL collocation points in each mesh element are sufficient to compute responses with good accuracy when compared to analytical solutions and to classical rigid density body approaches. The SEM is also much faster than the semi-analytical techniques of Blakely, with an acceleration factor larger than one order of magnitude. We have then shown an application to the western Pyrenees. With this new approach, it is straightforward to account for topography (here at the $1 \mathrm{mn}$ ETOPO1 resolution), Earth curvature as well as oceans. We have found that the Nafe-Drake law for scaling seismic pressure wave speed to density seems more accurate than Birch scaling for developing an a priori density anomaly model, at least in the western Pyrenees. A depth-weighting function leads to a better preconditioning of the gravity sensitivity matrix. This is an important ingredient of density tomography in order to have a more balanced sensitivity to density perturbations as a function of depth. In future work we plan to include more constraints in the a priori model, for instance by introducing additional constraints to account for sedimentary basins.

\section{ACKNOWLEDGEMENTS}

We thank the CALMIP supercomputing mesocentre of Toulouse, France, for their support through project P1138 on the EOS computer, and the TGCC/GENCI supercomputing centre through project \#gen6351 on the CURIE supercomputer. We are also grateful to the CNES granted projects \# 2015 and \# 2016-TOSCA/GET-GRAVI-GOCE for their financial support. This study was also supported by the Orogen research project, a tripartite academic-industry collaboration between CNRS, BRGM and Total.

\section{REFERENCES}

Amante, C. \& Eakins, B.W., 2009. ETOPO1 1 Arc-Minute Global Relief Model: Procedures, Data Sources and Analysis, NOAA Technical Memorandum NESDIS NGDC-24, 19, National Geophysical Data Center, NOAA.

Barnett, C.T., 1976. Theoretical modeling of the magnetic and gravitational fields of an arbitrarily shaped three dimensional body, Geophysics, 41(6), $1353-1364$.
Bhattacharyya, B.K., 1964. Magnetic anomalies due to prism shaped bodies with arbitrary polarization, Geophysics, 29(4), 517-531.

Bhattacharyya, B.K., 1980. A generalized multibody model for inversion of magnetic anomalies, Geophysics, 45, 255-270.

Birch, F., 1960. The velocity of compressional waves in rocks to 10 kilobars, Part 1, J. geophys. Res., 65(4), 1083-1102.

Birch, F., 1961. The velocity of compressional waves in rocks to 10 kilobars, Part 2, J. geophys. Res., 66(7), 2199-2224. 
Blakely, R.J., 1995. Potential Theory in Gravity and Magnetic Applications, Cambridge Univ. Press.

Brocher, T., 2005. Empirical relations between elastic wavespeeds and density in the Earth's crust, Bull. seism. Soc. Am., 95(6), 2081-2092.

Calcagno, P., Chilès, J., Courrioux, G. \& Guillen, A., 2008. Geological modelling from field data and geological knowledge. Part I. Modelling method coupling 3D potential-field interpolation and geological rules, Phys. Earth planet. Inter., 171, 147-157.

Casenave, F., Métivier, L., Pajot-Métivier, G. \& Panet, I., 2016. Fast computation of general forward gravitation problems, J. Geod., 90(7), 655-675.

Chakravarthi, V., Raghuram, H. \& Singh, S., 2002. 3D forward gravity modeling of basement interfaces above which the density contrast varies continuously with depth, Comput. Geosci., 28(1), 53-57.

Chevrot, S., Martin, R. \& Komatitsch, D., 2012. Optimized discrete wavelet transforms in the cubed-sphere with the lifting scheme-implications for global finite-frequency tomography, Geophys. J. Int., 191(3), 1391-1402.

Chevrot, S., Villaseñor, A., Sylvander, M. \& the PYROPE Team, 2014. High-resolution imaging of the Pyrenees and Massif Central from the data of the PYROPE and IBERARRAY portable array deployments, $J$. geophys. Res., 119(8), 6399-6420.

Chevrot, S., Sylvander, M., Diaz, J., Ruiz, M., Paul, A. \& the PYROPE Working group, 2015. The Pyrenean architecture as revealed by teleseismic P-to-S converted waves recorded along two dense transects, Geophys. J. Int., 200, 1096-1107.

Coggon, J., 1976. Pore pressure and poroelasticity effects in Coulomb stress analysis of earthquake interaction, Geoexploration, 114, 93-105.

Cohen, A., Daubechies, I. \& Fauveau, J., 1992. Biorthogonal bases of compactly supported wavelets, Commun. Pure Appl. Math., 45(5), 485-560.

Dubey, C.P. \& Tiwari, V.M., 2016. Computation of the gravity field and its gradient: Some applications, Comput. Geosci., 88, 83-96.

Dubey, C.P., Gotze, H., Schmidt, S. \& Tiwari, V., 2014. A 3D model of the Wathlingen salt dome in the Northwest German basin from joint modeling of gravity, gravity gradient, and curvature, Interpretation, 2(4), $1-13$.

Fichtner, A., 2010. Full Seismic Waveform Modelling and Inversion, Advances in Geophysical and Environmental Mechanics and Mathematics, Springer-Verlag, $343 \mathrm{pp}$.

Gardner, G.H.F., Gardner, L.W. \& Gregory, A.R., 1974. Formation velocity and density - the diagnostic basics for stratigraphic traps, Geophysics, 39(6), 770-780.

Gotze, H. \& Lahmeyer, B., 1988. Application of three-dimensional interactive modeling in gravity and magnetics, Geophysics, 53(8), 1096-1108.

Grandjean, G., 1994. Étude des structures crustales dans une portion de chaîne et de leurs relations avec les bassins sédimentaires, application aux Pyrénées occidentales, Bull. Centre Rech. Expl. Prod. Elf-Aquitaine, 18, 391-419.

Guillen, A., Calcagno, P., Courrioux, G., Joly, A. \& Ledru, P., 2008. Geological modelling from field data and geological knowledge, Part II modelling validation using gravity and magnetic data inversion, Phys. Earth planet. Inter., 171, 158-169.

Hansen, P., 2001. The L-curve and its use in the numerical treatment of inverse problems, in Computational Inverse Problems in Electrocardiology, Advances in Computational Bioengineering, vol. 5, pp. 119-142, ed. Johnston, P., WIT Press.

Komatitsch, D. \& Tromp, J., 1999. Introduction to the spectral-element method for 3-D seismic wave propagation, Geophys. J. Int., 139(3), 806822.

Lailly, P., 1983. The seismic inverse problem as a sequence of before stack migrations, in Expanded Abstracts, pp. 206-220, SIAM.

Lajaunie, C., Courrioux, G. \& Manuel, L., 1997. Foliation fields and 3D cartography in geology: principles of a method based on potential interpolation, Math. Geol., 29(4), 571-584.

Li, Y. \& Oldenburg, D.W., 1998. 3-D inversion of gravity data, Geophysics, 63(1), 109-119.
Li, Y. \& Oldenburg, D.W., 2003. Fast inversion of large-scale magnetic data using wavelet transforms and a logarithmic barrier method, Geophys. $J$. Int., 152, 251-265.

Ludwig, W., Nafe, J. \& Drake, C., 1970. Seismic Refraction, in The Sea, vol. 4, ed. Maxwell, A.E., Wiley-Interscience.

Martin, R., Monteiller, V., Komatitsch, D., Perrouty, S., Jessell, M., Bonvalot, S. \& Lindsay, M., 2013. Gravity inversion using wavelet-based compression on parallel hybrid CPU/GPU systems: application to southwest Ghana, Geophys. J. Int., 195(3), 1594-1619.

Monteiller, V., Chevrot, S., Komatitsch, D. \& Fuji, N., 2013. A hybrid method to compute short-period synthetic seismograms of teleseismic body waves in a 3-D regional model, Geophys. J. Int., 192, 230-247.

Monteiller, V., Chevrot, S., Komatitsch, D. \& Wang, Y., 2015. Threedimensional full waveform inversion of short-period teleseismic wavefields based upon the SEM-DSM hybrid method, Geophys. J. Int., 202, 811-827.

Nagihara, S. \& Hall, S.A., 2001. Three-dimensional gravity inversion using simulated annealing: constraints on the diapiric roots of allochthonous salt structures, Geophysics, 66(5), 1438-1449.

Nagy, D., 1966. The gravitational attraction of a right rectangular prism, Geophysics, 31(2), 362-371.

Nagy, D., Papp, G. \& Benedek, J., 2000. The gravitational potential and its derivatives for the prism, J. Geod., 74(7-8), 552-560.

Nava-Flores, M., Ortiz-Aleman, C., del Castillo, M. G.O., UrrutiaFucugauchi, J., Rodriguez-Castellanos, A., Couder-Castañeda, C. \& Trujillo-Alcantar, A., 2016. 3D gravity modeling of complex salt features in the southern gulf of Mexico, Int. J. Geophys., 1, 1-16.

Okabe, M., 1979. Analytical expressions for gravity anomalies due to homogeneous polyhedral bodies and translations into magnetic anomalies, Geophysics, 44(4), 730-741.

Paige, C.C. \& Saunders, M.A., 1982. LSQR: An algorithm for sparse linear equations and sparse least squares, ACM Trans. Math. Softw., 8(1), $43-71$.

Perrouty, S., Aillères, L., Jessell, M., Baratoux, L. \& Bourassa, Y., 2012. Revised Eburnean geodynamic evolution of the gold-rich southern Ashanti belt, Ghana, with new field and geophysical evidence of pre-Tarkwaian deformations, Precambrian Res., 204-205, 12-39.

Peter, D. et al., 2011. Forward and adjoint simulations of seismic wave propagation on fully unstructured hexahedral meshes, Geophys. J. Int., 186(2), 721-739.

Pilkington, M., 1997. 3-D magnetic imaging using conjugate gradients, Geophysics, 62(4), 1132-1142.

Pilkington, M. \& Hildebrand, A., 2000. Three-dimensional magnetic imaging of the Chicxulub crater, J. geophys. Res., 105, 23 479-23 491.

Plouff, D., 1976. Gravity and magnetic fields of polygonal prisms and application to magnetic terrain corrections, Geophysics, 41(4), 727-741.

Pohánka, V., 1988. Optimum expression for computation of the gravity field of a homogeneous polyhedral body, Geophys. Prospect., 36(7), 733-751.

René, R.M., 1986. Gravity inversion using open, reject, and 'shape-ofanomaly' fill criteria, Geophysics, 51(4), 988-994.

Roy, L., Sen, M.K., Blankenship, D.D., Stoffa, P.L. \& Richter, T.G., 2005. Inversion and uncertainty estimation of gravity data using simulated annealing: An application over lake Vostok, east Antarctica, Geophysics, 70(1), J1-J12.

Talwani, M., 1965. Computation with the help of a digital computer of magnetic anomalies caused by bodies of arbitrary shape, Geophysics, 30(5), 797-817.

Talwani, M. \& Ewing, M., 1960. Rapid computation of gravitational attraction of three-dimensional bodies of arbitrary shape, Geophysics, 25(1), 203-225.

Talwani, M., Worzel, J.L. \& Landisman, M., 1959. Rapid computation for two-dimensional bodies with application to the Mendocino submarine fracture zone, J. geophys. Res., 64, 49-59.

Tarantola, A., 1984. Inversion of seismic reflection data in the acoustic approximation, Geophysics, 49, 1259-1266.

Tarantola, A. \& Valette, B., 1982. Generalized nonlinear inverse problems solved using the least squares criterion, Rev. Geophys. Space Phys., 20, 219-232. 
Tromp, J., Tape, C. \& Liu, Q., 2005. Seismic tomography, adjoint methods, time reversal, and banana-doughnut kernels, Geophys. J. Int., 160, 195-216.

Tromp, J., Komatitsch, D. \& Liu, Q., 2008. Spectral-element and adjoint methods in seismology, Commun. Comput. Phys., 3(1), 1-32.

Tsoulis, D., 2012. Analytical computation of the full gravity tensor of a homogeneous arbitrarily shaped polyhedral source using line integrals, Geophysics, 77(2), F1-F11.

Uieda, L. \& Barbosa, V.C.F., 2012a. Robust 3D gravity gradient inversion by planting anomalous densities, Geophysics, 77(4), G55G66.

Uieda, L. \& Barbosa, V. C.F., 2012b. Use of shape-of-anomaly data misfit in 3D inversion by planting anomalous densities, in SEG Technical Program Expanded Abstracts 2012, vol. 4, pp. 1-6, SEG.

Uieda, L., Ussami, N. \& Braitenberg, C., 2010. Computation of the gravity gradient tensor due to topographic masses using tesseroids, EOS, Trans. Am. geophys. Un., 91(26), Meeting of the Americas Supplement, Abstract G22-A04.
Uieda, L., Bomfimi, E., Braitenberg, C. \& Molina, E., 2011. Optimal forward calculation method of the Marussi tensor due to a geologic structure at GOCE height, in Proceedings of the 4th International GOCE User Workshop, Munich, Germany.

Uieda, L., Barbosa, V.C. \& Braitenberg, C., 2016. Tesseroids: forwardmodeling gravitational fields in spherical coordinates, Geophysics, 81(5), F41-F48.

Virieux, J. \& Operto, S., 2009. An overview of full-waveform inversion in exploration geophysics, Geophysics, 74(6), WCC1-WCC26.

Wang, Y. et al., 2016. The deep roots of the western Pyrenees revealed by full waveform inversion of teleseismic P-waves, Geology, 44(6), 475-478.

Yao, L. \& Changli, Y., 2007. Forward modeling of gravity, gravity gradients and magnetic anomalies due to complex bodies, J. Chin. Univ. Geosci., 18(3), 280-286.

Zhang, J., Wang, C., Shiz, Y., Cai, Y., Chi, W., Dreger, D., Cheng, W. \& Yuan, Y., 2004. Three-dimensional crustal structure in central Taiwan from gravity inversion with a parallel genetic algorithm, Geophysics, 69(4), 917-924. 